\title{
Forced and Free Intra-Seasonal Variability Over the South Asian Monsoon Region Simulated by 10 AGCMs
}

\author{
MAN LI C. WU AND SIEGFRIED SCHUBERT \\ Data Assimilation Office, Goddard Space Flight Center, Greenbelt, MD, U.S.A.
}

\begin{abstract}
IN-SIK KANG
School of Earth and Environmental Sciences, Seoul National University, Seoul, Korea

and

DUANE WALISER

Institute for Terrestrial and Planetary Atmospheres, State University of New York, Stony Brook, New York, U.S.A.
\end{abstract}

23 August 2001

Submitted to Journal of Climate

Corresponding author: Man Li C. Wu, Data Assimilation Office, Goddard Space Flight Center, Greenbelt, MD, 20771 Email: mwu@dao.gsfc.nasa.gov TEL: 301 614-6249 


\begin{abstract}
This study examines intra-seasonal (20-70 day) variability in the South Asian monsoon region during $1997 / 98$ in ensembles of 10 simulations with 10 different atmospheric general circulation models. The 10 ensemble members for each model are forced with the same observed weekly sea surface temperature (SST) but differ from each other in that they are started from different initial atmospheric conditions.
\end{abstract}

The results show considerable differences between the models in the simulated 20-70 day variability, ranging from much weaker to much stronger than the observed. A key result is that the models do produce, to varying degrees, a response to the imposed weekly SST. The forced variability tends to be largest in the Indian and western Pacific Oceans where, for some models, it accounts for more than 1/4 of the 20-70 day intra-seasonal variability in the upper level velocity potential during these two years.

A case study of a strong observed MJO event shows that the models produce an ensemble mean eastward propagating signal in the tropical precipitation field over the Indian Ocean and western Pacific, similar to that found in the observations. The associated forced 200 mb velocity potential anomalies are strongly phase locked with the precipitation anomalies, propagating slowly to the east (about $5 \mathrm{~m} / \mathrm{s}$ ) with a local zonal wave number two pattern that is generally consistent with the developing observed MJO. The simulated and observed events are, however, approximately in quadrature, with the simulated response 
leading by 5-10 days. The phase lag occurs because, in the observations, the positive SST anomalies develop upstream of the main convective center in the subsidence region of the MJO, while in the simulations, the forced component is in phase with the SST.

For all the models examined here, the intraseasonal variability is dominated by the free (intra-ensemble) component. The results of our case study show that the free variability has a predominately zonal wave number one pattern, and has propagation speeds (10$15 \mathrm{~m} / \mathrm{s}$ ) that are more typical of observed MJO behavior away from the convectively active regions. The free variability appears to be synchronized with the forced response, at least, during the strong event examined here.

The results of this study support the idea that coupling with SSTs plays an important, though probably not dominant, role in the MJO. The magnitude of the atmospheric response to the SST appears to be in the range of $15 \%-30 \%$ of the $20-70$ day variability over much of the tropical eastern Indian and western Pacific Oceans. The results also highlight the need to use caution when interpreting atmospheric model simulations in which the prescribed SST resolve MJO time scales. 


\section{Introduction}

It is now well established that latent heat flux anomalies associated with the Madden Julian Oscillation (MJO) play an important role in the development of intraseasonal sea surface temperature (SST) anomalies in the Indian and western Pacific Ocean (Krishnamurti et al. 1988; Zhang and McPhaden 1995). Recent studies (e.g. Zhang 1996; Hendon and Glick 1997; Lau and Sui 1997; Sperber et al. 1997; Shinoda et al. (1998); Jones et. al. 1998) suggest that both MJO-induced surface evaporative and radiative flux anomalies contribute to intra-seasonal anomalies in SST. Hendon and Glick (1997) show that there are differences between the ocean basins, in that the Indian Ocean SST anomalies are primarily driven by surface insolation anomalies associated with convection, while the western Pacific SST anomalies are driven by both surface evaporation and insolation anomalies. Jones et al. (1998) provide a general picture of the MJO/SST relationship in which clear skies and reduced surface winds ahead of the convection anomaly, result in an increase in surface net shortwave radiation and decreased surface latent heat fluxes that favor positive SST anomalies. As the convection moves eastward over the warmer SST, the increased cloudiness and enhanced surface westerlies lead to reduced surface shortwave radiation and enhanced surface evaporation that favor negative SST anomalies.

Wang and Xie (1998) used a simplified linear coupled ocean-atmospheric model to carry out a theoretical analysis of the impact of SST feedback on the MJO. They found that the coupling produces SST anomalies that lead the convective anomalies and act to destabilize 
the atmospheric moist Kelvin wave and reduce its phase speed to observed values. Flatau et al (1997) investigated the impact of SST on the simulated MJO in an aquaplanet atmospheric general circulation model (AGCM) with a simple empirical representation of an ocean mixed layer. They found that the coupled model produced a stronger and slower MJO. They suggest that this occurred as a result of warmer SSTs to the east of the convection that acted to destabilize the atmosphere by increasing the moist static energy. Waliser et al. (1999) show that, in another AGCM coupled to a slab ocean model, an improved simulation of the MJO occurs compared with simulations with the same model that used prescribed SST. They also attributed the improvements in the simulations to feedbacks with the SSTs. They show, however, that positive SST anomalies forced by latent heating and to a lesser extent insolation anomalies to the east of the convection act to reinforce meridional convergence associated with the wave-CISK mechanism operating in the AGCM. Hendon et al. (2000) investigated the impact of air-sea coupling associated with the MJO in yet another AGCM coupled to a comprehensive ocean mixed layer model. That study found little impact of the coupling on the simulated MJO and showed that this was primarily due to deficiencies in the AGCM's latent heat flux that did not allow the formation of coherent SST anomalies.

A number of studies have examined the impact of SSTs on the interannual variation in MJO activity. Both observational (e.g. Hendon et al. 1999) and AGCM (e.g. Slingo et al. 1995; Gualdi et al. 1999) studies forced with monthly SSTs find generally only a weak relationship with SST. In particular, they find that while there is some impact of the SST 
on the spatial distribution of the MJO activity, the overall level of activity is largely uncorrelated with SST on interannual time scales. In a related study, Waliser et al. (2001) examined simulations with an AGCM forced with observed monthly SST in which the atmosphere was coupled to a weakly interacting slab ocean mixed layer. That study also found only a weak relationship with SST, although there was evidence of reduced Northern Hemisphere summer tropical intraseasonal variability during the extreme warm (El Nino) events.

Recently, Schubert and $\mathrm{Wu}$ (2001) found that, in an ensemble of AGCM runs with prescribed weekly SSTs, a significant forced "MJO-like" response occurred in the simulations during those times when strong MJO events occurred in the observations. This was interpreted as evidence of a potentially important feedback of the intraseasonal SST variations on the MJO. The current study extends the Schubert and Wu analysis by carrying out a more detailed analysis of the response to SST in ensembles of simulations with 10 different AGCMs. The model runs were carried out as part of the Asian-Australian Monsoon AGCM inter-comparison project sponsored by the World Climate Research Program/Climate Variability and Predictability (CLIVAR) Asian-Australian Monsoon panel (Kang et al. 2001).

Section 2 describes the simulated and observed data and outlines the diagnostics and processing steps used in the analysis. The results are presented in section 3 . Section $3 a$ describes the simulated and the observed intra-seasonal variability for $1997 / 98$. This 
includes the partition of the simulated variance into a forced and free component. Section $3 \mathrm{~b}$ examines the link between SST and the MJO for a particularly strong observed MJO event. Section $3 c$ describes the composite MJO and its links to SST and precipitation based on 17 years of National Centers for Environmental Prediction/National Center for Atmospheric Research (NCEP/NCAR) reanalysis and Global Precipitation Climatology Project (GPCP) precipitation observations. The discussion and conclusions are given in section 4 .

\section{Data and Analysis Methods}

\section{a) Model simulations}

The focus of the analysis is on the two-year 10-member ensemble simulations from 10 different AGCMs made available through the CLIVAR/GCM Monsoon Inter-comparison project. The AGCM simulations used here are from COLA (USA), DNM (Russia), GSFC (GEOS, USA), GSFC/SUNY(GLA, USA), GFDL (USA), IAP (China), IITM (India), MRI( Japan), NCAR (USA), and SNU (Korea). The details of the inter-comparison project and the description of the participated models can be found in Kang et al. (2001).

The 10-member ensemble AGCM simulations were performed for the period 1 September 1996 through 31 August 1998. The 10 ensemble members differ only in the initial atmospheric conditions. The SSTs are prescribed from the weekly SST data of Reynolds 
and Smith (1994). In addition to the $1997 / 98$ ensembles, the models were run for the period 1979-98 with prescribed observed monthly SSTs (see Kang et al. 2001). These longer runs were used to produce, for each model, a 5-day average (pentad) climatology that serves as a reference for analyzing the $1997 / 98$ time period. The basic model variables used here are the precipitation and the $200 \mathrm{mb}$ and $850 \mathrm{mb}$ winds. All GCM data were converted to a common spatial resolution of $2.0^{\circ}$ latitude $\times 2.5^{\circ}$ longitude, although the spatial resolution of the models varies from rhomboidal truncation at wavenumber 15 to triangular truncation at wavenumber 42 . The velocity potential and stream function fields were computed from the winds at the common resolution.

\section{b) Observed Data}

The atmospheric circulation and surface fluxes are obtained from the NCEP/NCAR reanalysis (Kalnay et al. 1996). In section 3a and 3b, we use the 1997/98 NCEP/NCAR $850 \mathrm{mb}$ and $200 \mathrm{mb}$ winds to compare with the model simulations for that time period. In section 3c, we analyze a longer record (1982-99) of NCEP/NCAR winds and surface latent heat and net shortwave fluxes to help clarify the link between the MJO and sea surface temperatures. The precipitation data consist of two GPCP products with sub-monthly temporal resolution. The first is a daily, $1^{\circ}$ horizontal resolution, multi-satellite precipitation data set available beginning in January 1997 (Huffman et al. 2001). The second is a 5-day averaged (pentad) precipitation data set available beginning in January 1979 (Xie and Arkin 1997). The pentad data were produced by merging several kinds of 
precipitation data including gauge observations and estimates inferred from infrared radiation (IR), outgoing long wave radiation (OLR), microwave sounding unit (MSU) and Special Sensor Microwave Imager (SSM/I) satellite observations.

\section{c) Data Processing}

We took several steps to isolate the intra-seasonal variability in both the models and the observations. For the reanalysis, pentad anomalies are computed from the 1982-98 pentad climatology. For the simulations, pentad anomalies were computed from each model's pentad climatology described earlier. Unless noted otherwise, all anomalies are filtered to retain only the time scales between 20-70 days. The filter is a symmetric, 4-pole, low-pass tangent-butterworth filter described in Oppenheim and Schafer (1975). The filter is applied twice, first retaining time scales longer than 20 days and then retaining time scales longer than 70 days. The bandpass data are obtain by subtracting the two filtered data sets. In our calculations, the end point effect is reduced by extending the ends of the series by duplicating the beginning and ending values.

\section{i. Forced and free variability}

For the simulations, the total variance of a quantity $(x)$ is divided into forced (interensemble) and free (intra-ensemble) components. This terminology is consistent with the idea that the ensemble mean of each model must be forced by the specified boundary 
conditions (SST anomalies), while the variations about each model's ensemble mean is uncorrelated with the SST, and therefore must be internally generated. As we shall see, the latter are not strictly free oscillations in that the intra-ensemble variability may be synchronized in time by the SST. An unbiased estimate of the inter-ensemble variance for a particular model is (see e.g., Rowell et al. 1995)

$$
s_{\beta}^{2}=\frac{n}{n-1}\left[(\bar{x}-[\bar{x}])^{2}\right]-\frac{1}{m} s_{\varepsilon}^{2}
$$

Here the over-bar denotes a mean over the $m=10$ ensemble members, the square brackets denote a mean over $\mathrm{n}$ independent time periods, and the subscript $\beta$ indicates that it is the forced variance. The second term on the right hand side of (1) is proportional to the intraensemble or free variance (indicated by subscript $\varepsilon$ ) and ensures that the estimate of the forced variance is unbiased. An unbiased estimate of the free variance is

$$
s_{\varepsilon}^{2}=\frac{m}{m-1}\left[\overline{(x-\bar{x})^{2}}\right]
$$

The total variance is defined as the sum of (1) and (2). The ratio of the forced variance to the total variance is define as

$$
P_{\beta}=\frac{s_{\beta}^{2}}{s_{\beta}^{2}+s_{\varepsilon}^{2}} .
$$


The ratio of the forced to free variance is defined as

$$
U=\frac{s_{\beta}^{2}}{s_{\varepsilon}^{2}} .
$$

Model-average estimates of $P_{\beta}$ and $U$ are obtained as in (3) and (4), except that $s_{\beta}^{2}$ and $s_{\mathcal{E}}^{2}$ are replaced by the averages of all the individual model forced and free variance estimates.

For the observations or reanalysis we have only one realization, so that the total variance is defined as

$$
s^{2}=\frac{n}{n-1}\left[(x-[x])^{2}\right]
$$

\section{ii. EOF and composite analysis}

In section 3c, we produce a composite picture of the evolution of the MJO. The composite is produced from the 17 years (1982-98) of NCEP/NCAR reanalysis data using the leading principal component of the filtered (20-70 day) $200 \mathrm{mb}$ velocity potential field as an index of the MJO. The principal components are the expansion coefficients of a complex empirical orthogonal function (CEOFs, e.g. Pfeffer et al 1990) decomposition. The CEOF 
decomposition provides an efficient spatial representation of a traveling disturbance such as the MJO. Details of the CEOF formulation may be found in Chang et al. (2001) and are not repeated here. The results of the CEOF decomposition lead to an expansion of a time series of fields $Z(x, y, t)$ that has the form

$$
Z(x, y, t)=\sum_{p=1}^{N} \alpha_{p}(t) \beta_{p}(x, y) \cos \left(\theta_{p}(x, y)-\phi_{p}(t)\right)
$$

where each component of (6) may be thought of as representing a wave with phase shape $\cos \left(\theta_{p}(x, y)\right)$ and time dependent phase $\phi_{p}(t)$, the amplitude of which is modulated in space by $\beta_{p}(x, y)$ and in time by $\alpha_{p}(t)$.

The composite fields described in section $3 c$ are obtained by averaging the fields for the appropriate phase of the first CEOF only during those times when the magnitude of $\alpha_{p}(t)$ exceeded one standard deviation.

\section{Results}

The results are divided into three subsections dealing with a) the basic $1997 / 98$ observed and simulated intra-seasonal variability, b) a case study of a strong MJO event during 1997 , and c) an analysis of the composite MJO for the period 1982-98.

We begin in subsection (a) by describing the intra-seasonal variability in both the model simulations and the observations for the period September 1996 through August 1998. The 
basic observed and simulated climatology and the $1997 / 98$ circulation and precipitation anomalies are documented in Kang et al. (2001) and Schubert and Wu (2001), and will not be repeated here. The filtered fields from the simulations are decomposed into forced and free components as described above. While our purpose here is not to highlight differences between the models, we do show how the models compare in the partition of the forced and free variance. For the most part, we focus on the averages over all model simulations allowing us to obtain greater confidence in the significance of the results. One difficulty with this model composite approach is that the models show such a wide range in the simulated intra-seasonal variability, that it is unlikely that they represent equally likely estimates of nature.

\section{a) Simulated Intra-seasonal Variability for $1997 / 98$}

The important role of intra-seasonal variations in the seasonal evolution of the rainfall in the southwest monsoon region is illustrated in Fig. 1 by the total rainfall (shaded) and the rainfall coming from MJO time scales (20-70 days) averaged over the longitudes spanning the Indian subcontinent $\left(75^{\circ} \mathrm{E}\right.$ to $\left.85^{\circ} \mathrm{E}\right)$. The observed rainfall (GPCP) is shown in Fig. 1a, and the rainfall obtained by averaging all the simulations is shown in Fig. 1b. The observations show a clear northward progression of the rainfall from the southern Hemisphere into the northern Hemisphere that tends to occur on the MJO time scales. For example, the May increase and late May and early June break in the monsoon rainfall during 1997 coincides with the positive and negative rainfall anomalies associated with the 
MJO time scales. The grand mean of the AGCM simulations (Fig. 1b) shows a similar though more gradual seasonal evolution. This is, to some extent, to be expected since the results are an average over many cases so that individual $\mathrm{MJO}$ events (to the extent that they are simulated by the models) are averaged out. Perhaps the most surprising aspect of Fig. 1b is that there is evidence of MJO variability in the model simulations that is not averaged out (the contours of 20-70 day variability shown in Fig. 1b), but is common to all (or at least many) of the $100 \mathrm{AGCM}$ simulations. This is consistent with the results of Schubert and Wu (2001) based on just one of the AGCMs included here (the GEOS model). The fact that we find such an ensemble mean MJO signal in the grand ensemble and model mean suggests that further study of the nature of this apparently forced variability is warranted.

Figure 2 shows a time-longitude diagram of the unfiltered $200 \mathrm{hPa}$ pentad velocity potential anomalies (VLPAs) averaged between $5^{\circ} \mathrm{N}$ and $10^{\circ} \mathrm{N}$. The NCEP/NCAR reanalysis shows evidence of substantial MJO activity especially during the first half of 1997 when there are well defined propagating anomalies. The late May and early June Indian monsoon break mentioned earlier is also evident here. During late 1997 and 1998 , we see a low frequency shift that appears to be the signature of the developing El NinoSouthern Oscillation (ENSO). There is also evidence of MJO variability during the spring and summer of 1998 (though with somewhat shorter time scales compared with 1997; see also Schubert and $\mathrm{Wu}, 2001$ ). The ensemble and model mean unfiltered pentad VLPAs (deviations from each models pentad climatology) are superimposed as contours in the left 
panel and repeated in color in the center panel. The three contour levels in the center panel indicate the regions where the ensemble mean values are significant at the 20,10 and $5 \%$ level, based on a t-test. The simulated VLPAs display significant MJO signals that occur at approximately the time when major events occurred in nature. The ensemble mean of the simulated events, however, are weaker than the anomalies found in the reanalysis. They also tend to lead, in fact, they tend to be in quadrature with, the observed anomalies. We will return to the issue of the phasing of the events in the next section. These results are again consistent with the results from the GEOS model reported in Schubert and Wu (2001), and suggest that the models are responding to the intraseasonal variability in the specified weekly SST observations.

The right panel of Fig. 2 shows the evolution of the 20-70 day filtered intra-ensemble or free variance, $s_{\varepsilon}^{2}$ (averaged over all models). This shows that enhanced free variability in the velocity potential tends to coincide with periods during which enhanced ensemble mean velocity potential anomalies occur. This is particularly evident in 1998 when there is a substantial increase in the free variability east of about $120^{\circ} \mathrm{W}$ in a region of positive ensemble mean anomalies (associated with ENSO-related shift in the Walker circulation). After June 1998 , there is also an increase in variability west of $120^{\circ} \mathrm{W}$ coinciding with the development of negative ensemble mean anomalies over the eastern Indian Ocean.

Figure 3 shows maps of the total 20-70 day filtered intra-seasonal variance simulated by each model and compares them with the results from the NCEP/NCAR reanalysis. We see 
that the models exhibit a wide range of variability. On one extreme is the IAP model which shows variability more than twice that of the reanalysis throughout the tropics. On the other end of the spectrum is the DNM model, which has variance about a factor of 10 less than that of the reanalysis. The GFDL model appears to be closest to the reanalysis showing variability that is similar to the reanalysis in both magnitude and spatial distribution.

We next partition the simulated filtered intra-seasonal variability into forced (variance of the ensemble mean) and free (intra-ensemble variance) components as described in Section 2ci. The results, shown in Fig. 4, indicate that for all the models the free variance dominates the intraseasonal filtered variability. All the models have forced variability that is largest over the eastern Indian Ocean and the western Pacific Ocean. There are, however, again substantial differences between the models in both the magnitude and in the partition of the forced and free variability. The SUNY/GLA model has the largest forced variability while the DNM model has the weakest. The large IAP model variability mentioned earlier is mostly in the free component. The partition of the variance is seen more clearly in Fig. 5, which shows, in the left panel, the ratio of the forced to total filtered variability $\left(P_{\beta}\right)$ and, in the right panel, the ratio of the forced to free filtered variability $(U)$. The SUNY/GLA and DNM models have the largest values of $P_{\beta}$ with values exceeding $30 \%$ over parts of the eastern Indian Ocean and/or the western Pacific Ocean. The MRI, NCAR and SNU models also have substantial fractions of the variability in the forced 
component with values of $P_{\beta}$ exceeding $20 \%$ over much of the western Pacific and Indian Oceans. The two models with the most realistic total variance (see Fig. 3) show considerable differences in $P_{\beta}$, with the GFDL model showing values that are generally less than $20 \%$, compared with more than $30 \%$ for the SUNY/GLA model. More than half of the models have signal (the forced response) to noise ratios $(U)$ that are greater than $30 \%$.

\section{b) A case study}

In this section, we examine in some detail a particular MJO event that occurred during the spring of 1997. Fig. 6 shows the time series of the simulated and reanalysis filtered $200 \mathrm{mb}$ velocity potential and the SST for a region in the tropical Indian Ocean. Here we have changed the sign on the VLPAs to show more clearly the phasing with the SST. All the time series show substantial intra-seasonal oscillations during the first half of 1997 . The reanalysis and simulated VLPAs appear to be synchronized with each other and with the SST anomalies though the exact phasing appears to change somewhat with time. The model simulations show VLPAs that are strongly coupled with the SST anomalies, with warm SST anomalies associated with negative VLPAs. The reanalysis velocity potential also shows a link to the SST though at times it is nearly in quadrature with the SST (e.g. during September/October and during April 1997), consist with the picture presented in earlier studies of clear skies (positive VLPAs) and reduced winds leading to a warmer ocean (e.g. Jones et al. 1998). To better understand these relationships we next focus on 
the MJO event that occurred during the time period February 12 through May 12, 1997. This event was chosen because it is one of the most pronounced oscillations to occur during these two years, and it is not immediately preceded by other large events which could introduce SST anomalies that persist into the next event. We therefore hope to obtain a clearer picture of the link between the development of SST anomalies and the MJO.

Figure 7 shows the filtered (20-70 day) SST anomalies (deviations from the 1982-98 climatology) averaged every 10-days. While the field is somewhat noisy, there is evidence, beginning in February, of eastward propagation of the cold anomalies from the Indian Ocean into the western Pacific. Also, the warm SST anomalies that develop over the western Indian Ocean at the beginning of March appear to propagate into the western Pacific in about 40 days.

The simulated and reanalysis filtered $200 \mathrm{mb}$ VLPAs for that period are shown in Fig. 8 . The simulated results (left panel) are the average over all ensemble members and models (the forced component). The simulations show a coherent time evolution, with a dipole structure developing over the Indian Ocean and Indonesia that has a spatial scale consistent with about a wave number two structure, which then strengthens and propagates slowly (about $5 \mathrm{~m} / \mathrm{s}$ ) to the east. A comparison with the SST anomalies in Fig. 7 shows that the development is roughly consistent with the SST in the sense that anomalous rising motion tends to coincide with warm SST anomalies and anomalous sinking motion tends to coincide with cold SST anomalies (see also Fig. 6). The reanalysis shows a qualitatively 
similar evolution, starting also as a wave number two dipole over the Indian Ocean and Indonesia and propagating to the east, though after about 30 days it evolves into a zonal wave one structure characteristic of the MJO. Fig. 8 also shows that the simulated and observed anomalies are not in phase, in fact they tend to be in quadrature, especially during the first part of the period, with the simulations leading the observed anomalies in the eastern hemisphere. In fact, if one were to shift the simulated anomalies about 10 days back (one panel in Fig. 8), the observed and simulated anomalies would be nearly in phase throughout much of the eastern hemisphere. This is quantified in Fig. 9, which shows the lag correlations between the simulated and observed anomalies. This shows that the largest positive correlations $(>0.90)$ occur over the Indian Ocean when the simulated anomalies lead the observed by 10 days. Over the western Pacific Ocean, the simulated anomalies lead by about 5 days. Since the anomalies oscillate in time, negative correlations tend to occur at opposite (positive) lags.

While the reanalysis shows the anomaly propagating around the globe, the simulated ensemble mean anomalies are largely confined to longitudes west of about $120^{\circ} \mathrm{W}$. As we shall see next, this reflects the strong link between the ensemble mean velocity potential and SST anomalies in the Indian and western Pacific Oceans. Note that the mean simulated anomalies are about a factor of three or four smaller than the reanalysis anomalies. This is presumably in part due to the averaging process: the anomalies from the reanalysis represent a single realization (including both forced and free components). Outside the convectively active region, the simulated variability is primarily free and the averaging 
tends to cancel out the anomalies from the various runs: we will discuss the nature of the free variability later in this section.

Figure 10 is the same as Fig. 8, except for the precipitation anomalies. The superimposed contours are a repeat of the VLPAs from Fig. 8. The results show a strong relationship between the precipitation and VLPAs, especially for the simulations. For example, in the beginning of March both the models and the reanalysis show enhanced precipitation south of the equator near the dateline and generally reduced precipitation to the west. Both the simulations and the model show a local zonal wave number two velocity potential "response" to the precipitation anomalies. Subsequently, the reanalysis VLPAs propagate eastward and appear to decouple from the rainfall anomalies, and develop into a wave number one structure. The mean simulated anomalies, on the other hand, remain in lock step with the precipitation anomalies. The simulated mean precipitation anomalies in the Indian Ocean and western tropical Pacific (left panel of Fig. 10) appear to be strongly linked to the SST anomalies (Fig. 7). The observed precipitation anomalies (right panel of Fig. 10), while similar to the simulated precipitation anomalies, show less of a direct spatial coherence with the SST anomalies, especially over the Indian Ocean.

While the previous results focused on the ensemble mean forced response to the SST, we can also examine the structure of the free (intra-ensemble) variability during the Feb 12May 12 time period. We accomplish this by computing the intra-ensemble variance and its time lag correlations. In particular, we compute the lag correlations between the free (intra- 
ensemble) fluctuations in the $200 \mathrm{mb}$ velocity potential at a base region (an average over the region $80^{\circ} \mathrm{E}-110^{\circ} \mathrm{E}, 15^{\circ} \mathrm{S}-10^{\circ} \mathrm{N}$ ) and a base time (the 10-day average of March 24-April 2), with the free fluctuations in the velocity potential at all grid points and other times. We compute an "all model" lag correlation by averaging the covariances and variances over all the models before dividing to compute a correlation.

The evolution of the intra-ensemble variance (left panel of Fig. 11) shows a general increase in time over Indonesia and the western Pacific through April 3, followed by a decline. Over Africa and the Indian Ocean, the variance is largest during April, whereas over South America it peaks at the beginning of March. There does not appear to be a strong correspondence between the evolution of the signal (Fig. 10) and the intra-ensemble variance in Fig. 11. The lag correlation results (right panel of Fig. 11) provide a picture of the spatial structure and evolution of the free variability that is different from that of the forced response shown in Fig. 8 (left panel). Instead of the slowly propagating, predominantly local wave number two pattern in the western hemisphere, the free variability is characterized by a faster propagating (about $10-15 \mathrm{~m} / \mathrm{s}$ ) zonal wave number one structure that is more reminiscent of the behavior of the observed MJO away from the convectively active regions of the western Pacific and Indian Oceans (c.f. right panel of Fig. 8). Note that the fact that we obtain a coherent lag correlation signal at all, suggests that the free variability must be to some extent synchronized with the forced response (though it could be of either sign). We have also computed the EOFs of the free variability for this time period (not shown), and found that the leading modes have a structure that is 
very similar to that shown in the right panel of Fig. 11. In those calculations, the EOFs were computed from the extended correlation matrix that includes all pentads between Feb 12 and May 12 of 1997.

The above results show that the free and forced variations have substantially different characteristics. The free variability is similar in structure and phase speed to the observed MJO away from the convectively active regions of the western Pacific and Indian Oceans. The forced response, on the other hand, is largely confined to the convectively active regions, and tends to lead the observed MJO by about ten days. This appears to reflect the fact that in nature, warm SST anomalies develop ahead of the MJO's main convective center, while the cold anomalies develop behind it. In the model simulations, the response is, however, in phase with the SST, and one might argue that this response is indicative of the feedback to the SST that occurs in nature on, for example, the leading edge of the convective center of the MJO. We next look in more detail at the observed behavior of the MJO and its links to SSTs and convective heating using 17 years (1982-98) of NCEP/NCAR reanalysis data and the GPCP pentad precipitation fields.

\section{c) The composite $\mathrm{MJO}$}

We begin by computing the complex EOFs (CEOFs, see section 2cii) of the filtered (20-70 days) $200 \mathrm{mb}$ VLPAs for all seasons for the period 1982-1998. The leading CEOF accounts for $3 / 4$ of the filtered variance and represents a traveling zonal wave number one 
disturbance. The results in Figs. 12-14 are displayed as composites, computed using the time series of the amplitude of the leading CEOF as an index and averaging according to the phase of the oscillation. Values are included in the composite only for those times when the amplitude of the CEOF exceeds one standard deviation. The zonal wave one structure and eastward propagation of the VLPAs shown in these Figures is consistent with previous analyses of the MJO (e.g. Weickmann et al 1985; Hendon and Salby 1994). The time scale of the oscillation is (again consistent with the MJO) predominantly between 30 60 days (see Chang et al. 2001), so that the panels in Figs. 12-13 show events that are approximately 5 days apart.

The left panel of Fig. 12 shows the composite evolution of the filtered SST anomalies associated with CEOF 1. The right panel of Fig. 12 shows the composite filtered $200 \mathrm{mb}$ velocity potential (contoured) and precipitation (shaded) anomalies. The SST anomalies show evidence of eastward propagation over the Indian Ocean and western Pacific warm pool as the CEOF cycles through the phases -180 to 0 degrees. In this region, the warmest SST tend to occur on the leading edge of the rising branch of the MJO. In the eastern Pacific the SST anomalies tend to be positive during the phases -180 to -135 and negative during the phases -90 and +90 to +180 . This suggests that the development of the MJO (or at least the convectively active Indian Ocean phase) is favored when warm SST occur in both the Indian and eastern Pacific Oceans .

The right panel of Fig. 12 shows that, as the rising branch of the MJO enters the Indian 
Ocean, the precipitation is enhanced on its leading edge (phases $-180^{\circ}$ to $-135^{\circ}$ ) Precipitation is reduced over the trailing region of the sinking branch over the Pacific warm pool. The maximum precipitation anomaly occurs at a phase of $-90^{\circ}$, when it is centered on the rising branch of the $\mathrm{MJO}$ near $90^{\circ} \mathrm{E}$ longitude. This is consistent with previous studies (e.g. Hendon and Salby 1994). At a phase of $-45^{\circ}$ the precipitation anomalies split, with two local maxima (one north and one south of the equator) on the trailing edge of the rising branch, and a single maximum on the leading edge. The northern precipitation anomaly subsequently moves north/north east over India, the Bay of Bengal, Indochina and the Pacific warm pool. This is reminiscent of the typical evolution of low frequency intraseasonal summer monsoon fluctuations in that region (e.g. Yasunari 1981; Wu et al. 1999). The split in the rainfall anomalies occurs in conjunction with (and appears to be a response to) a relatively strong Rossby wave response ( Gill 1980) in the western hemisphere. The Rossby wave response is clearly evident in Fig. 13, which shows the composite stream function anomalies at $850 \mathrm{mb}$ and $200 \mathrm{mb}$ (see, in particular, the stream function anomalies at phase $-90^{\circ}$ in Fig. 13). The northward movement of the rainfall over South Asia is associated with a similar northward displacement of the $850 \mathrm{mb}$ stream function anomalies between phases $-90^{\circ}$ to $+45^{\circ}$ (left panel of Fig. 13). Beyond a phase of $0^{\circ}$, the rising branch of the MJO becomes decoupled from the precipitation anomalies which themselves become weak. As the sinking branch enters the Indian Ocean (phase $>0^{\circ}$ ), the precipitation anomalies are approximately repeated but with the opposite sign to those during the phases $-180^{\circ}$ to $0^{\circ}$. 
Comparing the two panels of Fig. 12, we see that the correspondence between the precipitation and SST anomalies is rather complex in the observations. The positive precipitation anomalies develop just south of India in the presence of warm SST anomalies (top panels of Fig. 12). For the next 10-15 days the center of the warm precipitation anomalies moves very little (about $10^{\circ}$ longitude), while warm SST anomalies develop farther to the east over Indonesia and the Pacific warm pool. At phases $-45^{\circ}$ and $0^{\circ}$, after the precipitation anomalies have split (see above), the eastern component of the positive precipitation anomalies appear to be linked to (but occur just west of) very localized warm SST anomalies near $165^{\circ} \mathrm{E}$ longitude.

In Fig. 14, we summarize the relationships between the composite SST anomalies and several other fields using phase-longitude plots. The top left panel shows that positive SST anomalies tend to occur on the leading edge of (just east of) the main rising branch of the MJO in the Indian Ocean and western Pacific. Our results concerning the forcing of the SST anomalies by latent heat and short wave flux are generally consistent with previous studies. We find that the latent heat flux anomalies tend to lead the SST anomalies in both the Indian and western Pacific Oceans, with reduced (enhanced) latent heating leading warm (cold) SST anomalies (lower left panel of Fig. 14). The lag time is somewhat variable but tends to be about 5-10 days. Also, the net short wave flux anomalies are such that enhanced (reduced) short wave flux leads warm (cold) SST anomalies by 5-10 days (upper right panel of Fig. 14). It is, however, important to keep in mind that the reanalysis flux quantities are model-generated and therefore are subject to model bias errors. Shinoda 
et al. (1999), for example, show that while the NCEP/NCAR reanalysis latent heat fluxes are reasonable, the short wave fluxes are about half the amplitude of satellite-based estimates on intraseasonal time scales. In the eastern Pacific Ocean, there is no clear relationship between the SST and either the insolation or the latent heat flux anomalies. The lower right panel of Fig. 14 shows that the SST anomalies west of $180^{\circ}$ tend to lead the main precipitation anomalies supporting the results of Waliser et al (1999) and others, that suggest a feedback role for the SST in the development of the MJO. In the western Pacific Ocean the lag between the SST and precipitation anomalies appears to be about 10 days, while in the Indian Ocean the lag is about 5 days.

\section{Summary and Conclusions}

There is growing observational and theoretical evidence that the atmospheric MJO is part of a coupled atmospheric/ocean phenomena (e.g. Zhang 1996; Hendon and Glick 1997; Wang and Xie 1998; Jones et. al. 1998). This is bolstered by recent studies employing AGCMs coupled to mixed layer ocean models that note improved simulations of the MJO (Flatau et al. 1997; Waliser et al. 1999). The results are, however, model dependent and the degree of improvement (and possibly the mechanisms involved) appear to depend on the differing capabilities of the uncoupled AGCMs to simulate the MJO (Hendon et al. 2000).

This study took a somewhat different approach to addressing the MJO/SST coupling issue by examining whether AGCMs run with prescribed SSTs exhibit a significant atmospheric 
respond to the SST on intraseasonal time scales. In light of the strong model dependence of the results noted above, we employed ensembles of simulations produced by 10 different AGCMs. These runs were produced using observed weekly SSTs for the two-year period $1997 / 98$ as part of the Asian-Australian Monsoon AGCM inter-comparison project sponsored by the World Climate Research Program/CLIVAR Asian-Australian Monsoon panel (Kang et al. 2001).

The analysis involved decomposing the 20-70 day variability into forced (inter-ensemble) and free (intra-ensemble) components. The results showed considerable differences among the models in the representation of the $20-70$ day velocity potential variability at $200 \mathrm{mb}$, ranging from much weaker to much larger than the observed variance. A key result is that the models do produce, to varying degrees, an ensemble mean response to the imposed weekly SST. The forced variability in the models tends to be largest in the Indian and western Pacific Oceans, and occurs primarily during periods of strong observed MJO activity. For some models, the forced (inter-ensemble) variations account for more than $1 / 4$ of the 20-70 day intra-seasonal variability in the upper level velocity potential during these two years. .

A case study of a strong MJO event showed that the models produce an ensemble mean eastward propagating signal in the tropical precipitation field over the Indian Ocean and western Pacific, similar to that found for the MJO event in the observations. The associated forced $200 \mathrm{mb}$ VLPAs are strongly phase locked with the precipitation 
anomalies, propagating slowly to the east (about $5 \mathrm{~m} / \mathrm{s}$ ) with a local zonal wave number two pattern that is generally consistent with the developing MJO found in the observations. A comparison of the phases of the two phenomena, however, shows that they are approximately in quadrature, with the simulated response leading the observed by $5-10$ days. The phase lag occurs because, in the observations, the positive SST anomalies develop upstream of the main convective center in the subsidence region of the MJO, as a result of reduced latent heat flux and increased net surface insolation. In the simulations, the forced component is in phase with the SST so that the simulated convection and VLPAs lead those of the observations by 5-10 days.

For all the models examined here, the intraseasonal variability is dominated by the free (intra-ensemble) component. Increases in the magnitude of the free variability tend to coincide with periods during which the forced response is strongest. The results of our case study show that the free variability has a zonal wave number one pattern, and has propagation speeds $(10-15 \mathrm{~m} / \mathrm{s})$ that are faster than those of the forced component, and more typical of observed MJO behavior away from the convectively active regions. Furthermore, we found that the free variability appears to be synchronized with the forced response, at least, during the strong event studied here.

The extent to which the ensemble/model mean simulated response to prescribed SSTs provides a reasonable estimate of the magnitude of the true SST feed back on the MJO is unclear. It is of course possible that the SST feedback in the coupled problem is 
substantially different from the response to prescribed SSTs. In fact, the phase difference between the forced response in the models and the response estimated from the observations suggests this is may be the case. On the other hand, one could argue that the phase difference is not inconsistent with an SST feedback that occurs on the leading edge (and is a small fraction of) the main convective anomaly of the MJO. Nevertheless, we suggest that our analysis provides a reasonable estimate of the magnitude of the feedback in the coupled problem, subject to model errors as discussed below. This issue could be examined in future studies by comparing AGCM runs in which the same model is run in both coupled and uncoupled modes. The phase difference also suggests that one must be careful in the interpretation of tropical variability that exists in AGCM simulations with prescribed SST, when those SST contain time scales short enough to resolve the MJO.

Finally, the large range in the variability among the 10 models in both the relative $\left(P_{\beta}\right)$ and absolute value of the forced response shows that current convective schemes vary considerably in their sensitivity to SSTs, and indicates that the schemes have not yet converged on the correct response (see also Slingo et al. 1996). It is noteworthy that even for the two models with the most realistic intraseasonal variability (GFDL and SUNY/GLA), there are considerable differences in both the magnitude and distribution of the forced variability. Nevertheless, these results support the idea that coupling with SSTs plays an important, though probably not dominant, role in the MJO. The magnitude of the response of the atmosphere to SSTs appears to be in the range of $15 \%-30 \%$ of the total intraseasonal (20-70 day) variability over much of the tropical eastern Indian and western 


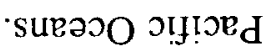




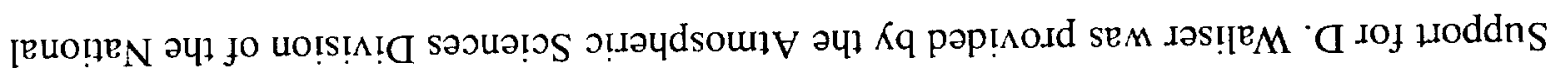

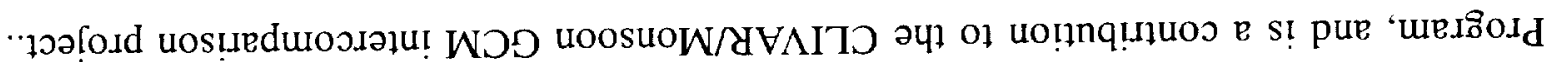

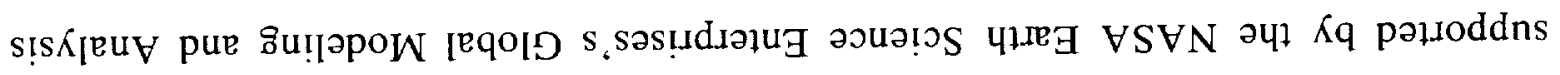

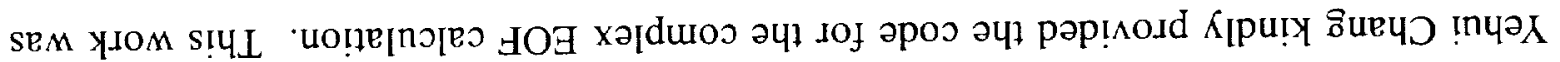




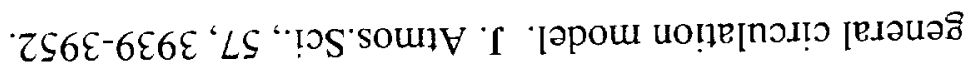

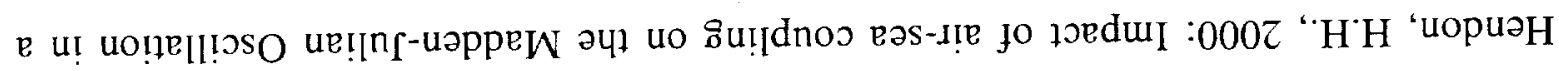

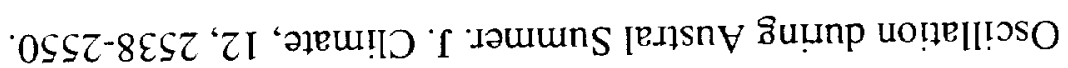

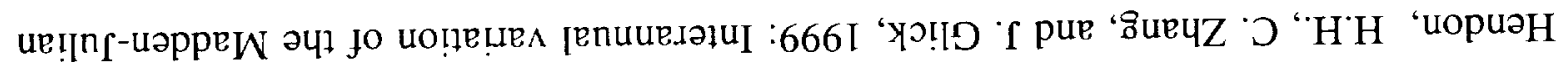

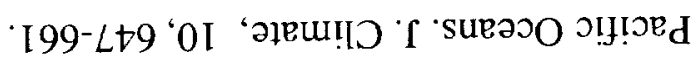

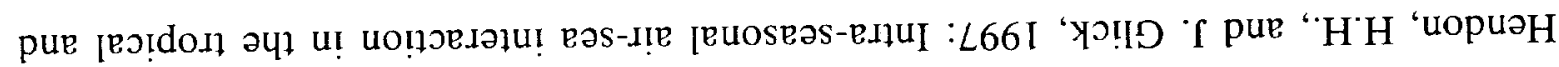
'LEZZ-SZZZ "IS "IOS' Sount

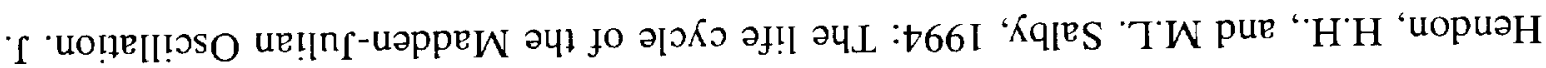

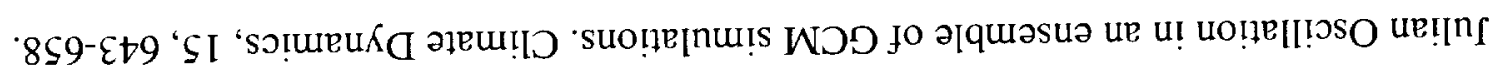

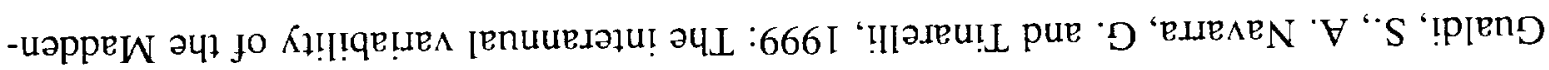

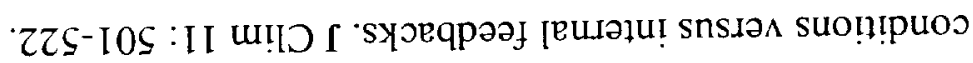

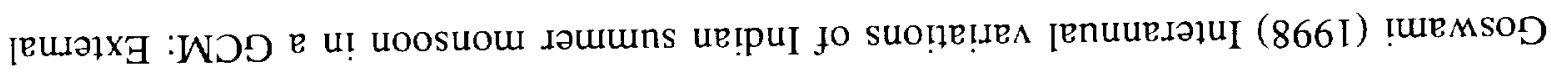

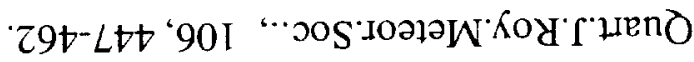

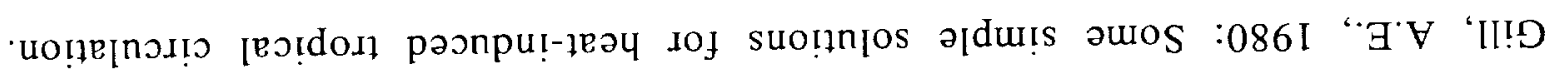

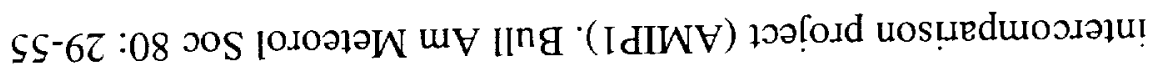

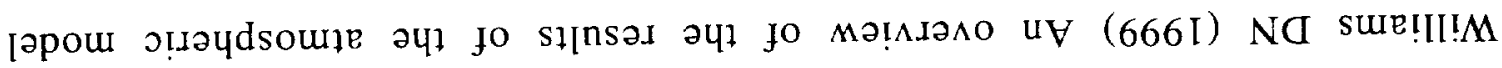
'

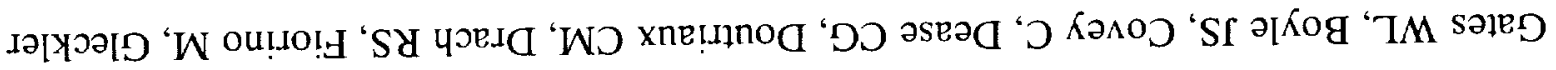

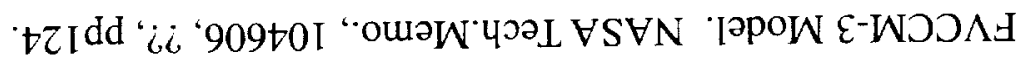

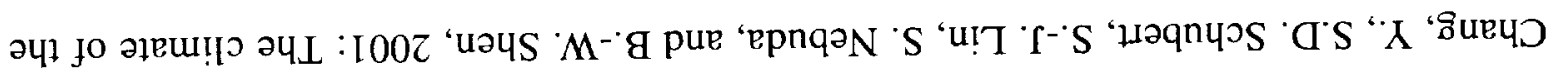




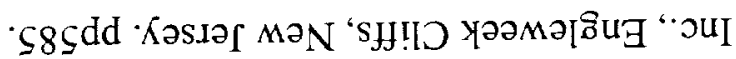

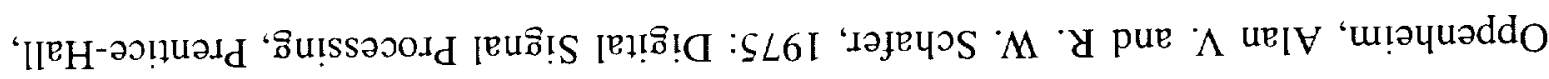

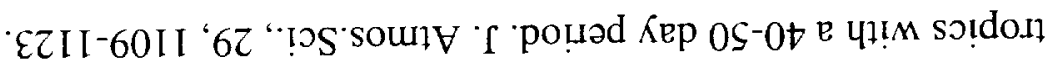

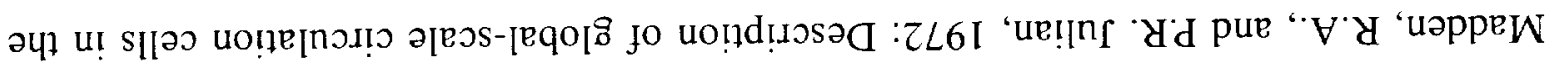

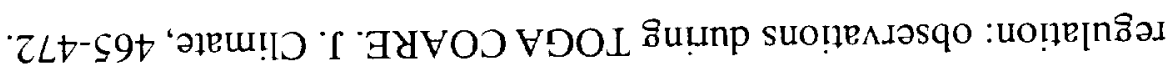

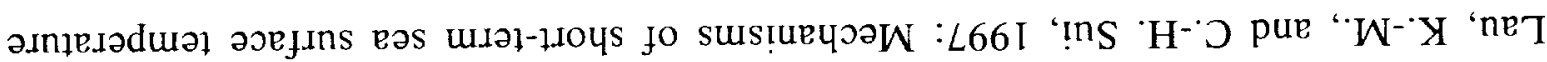

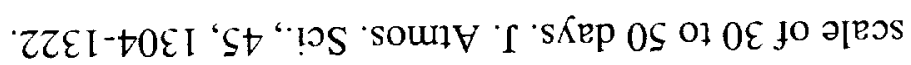

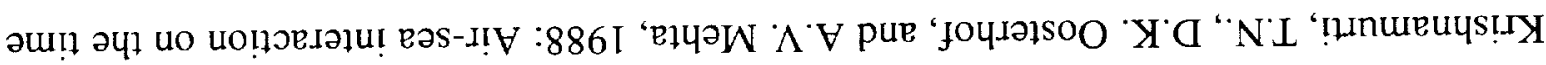

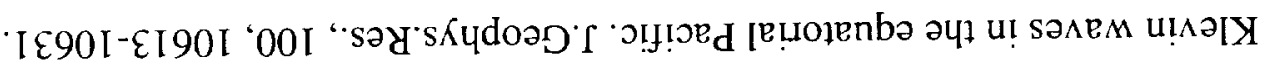

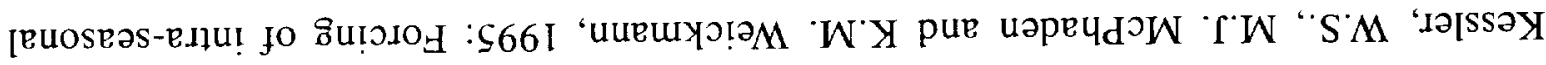

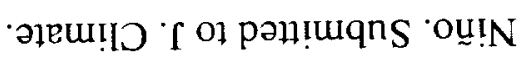

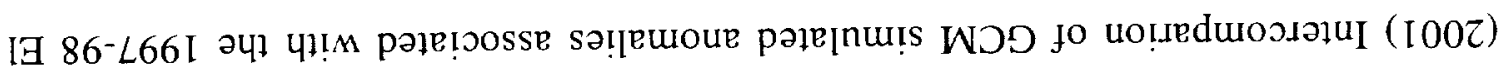

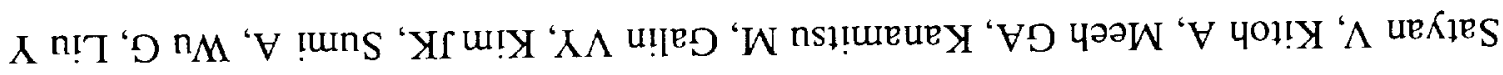

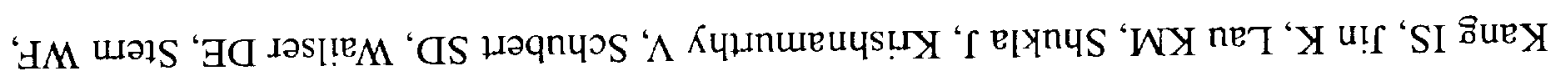

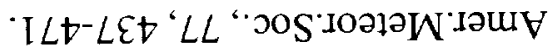

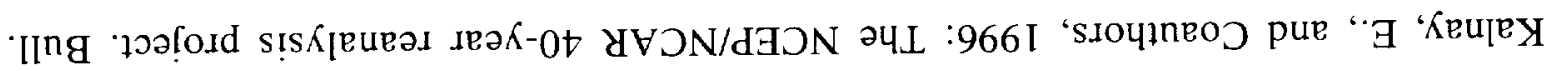
ZLOI-LSOI

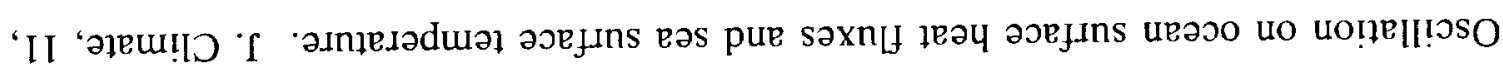

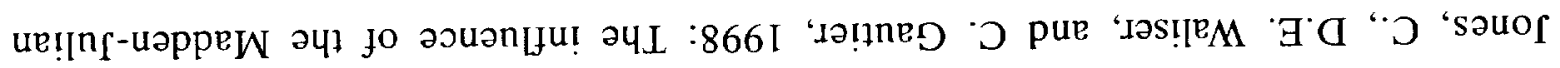

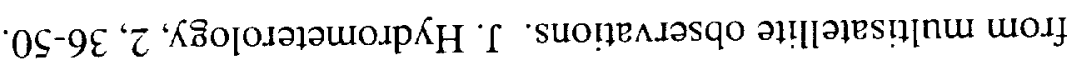

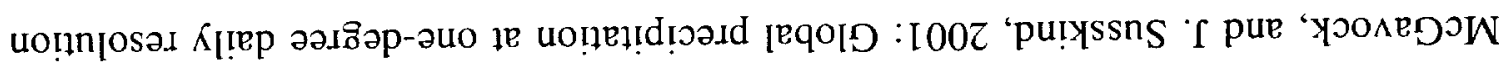

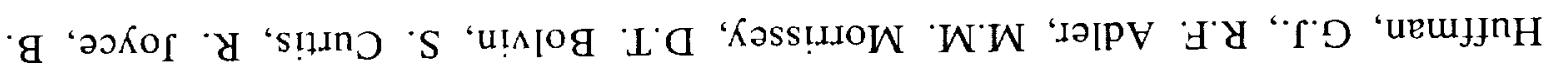




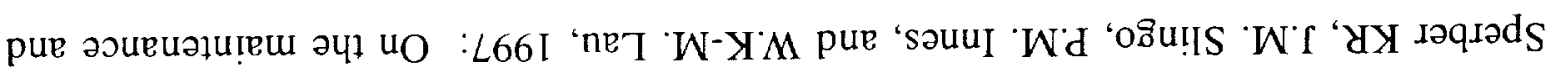

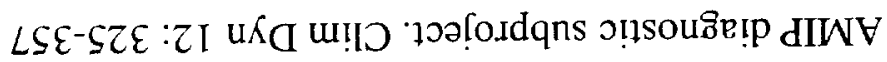

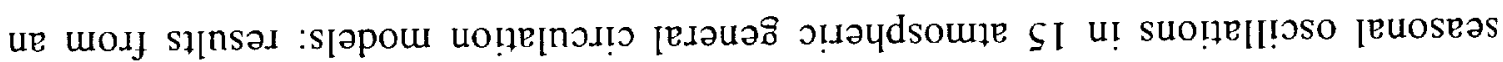

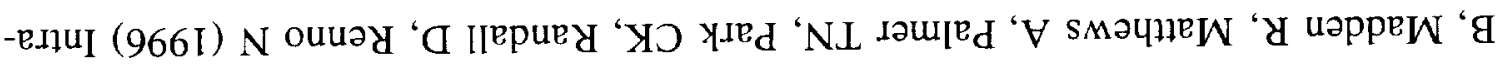

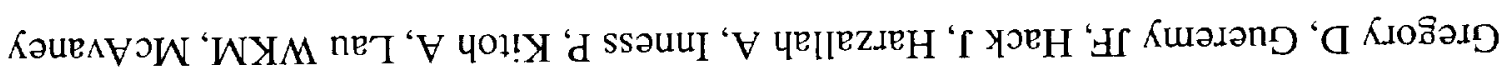

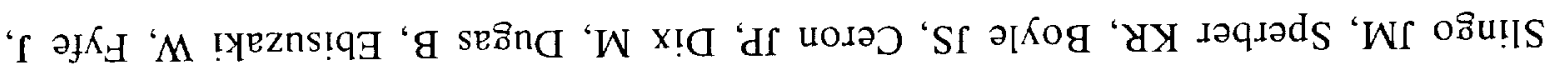

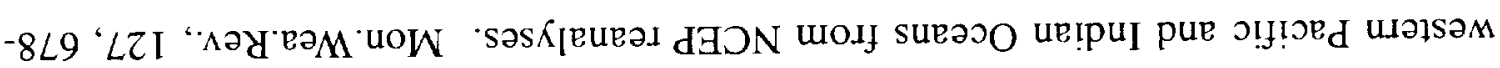

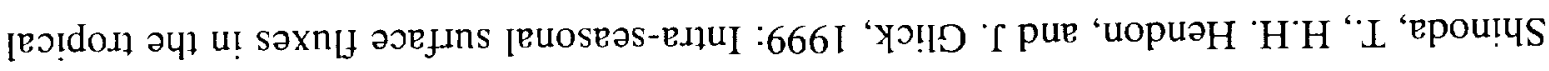

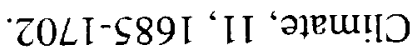

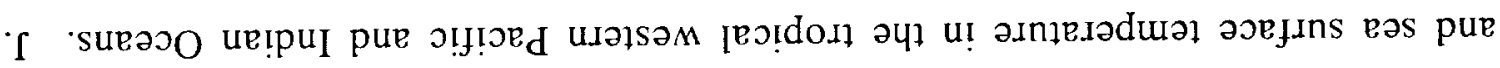

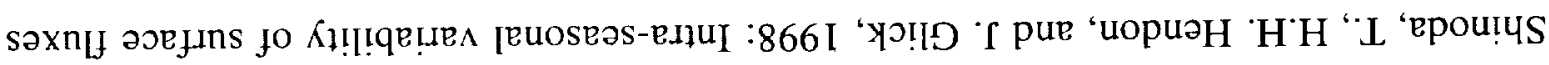

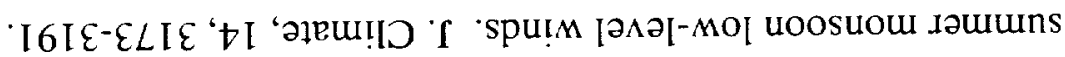

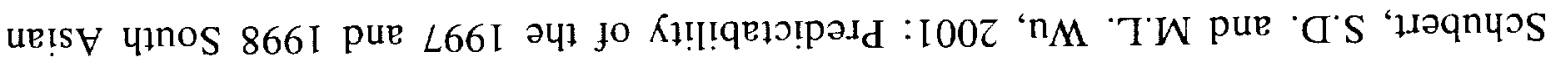
†0L-699 ‘ I I I “oо:

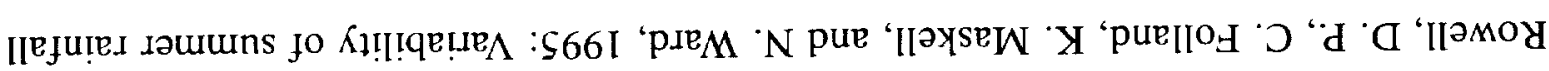

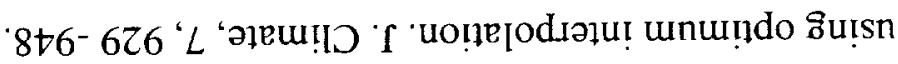

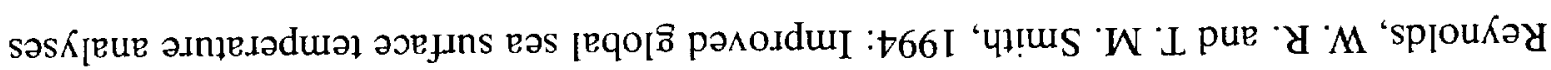

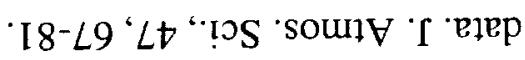

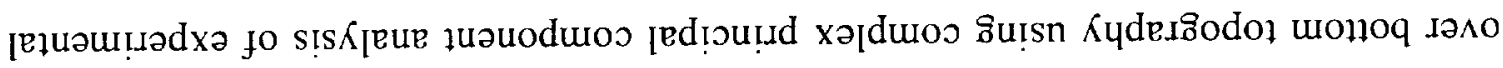

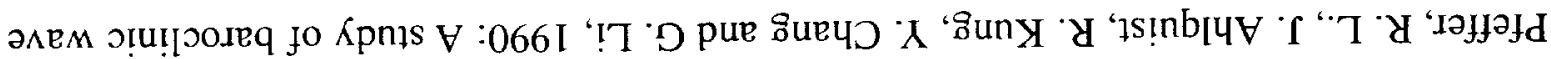




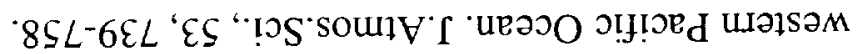

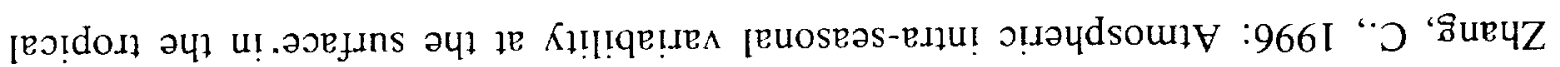
t

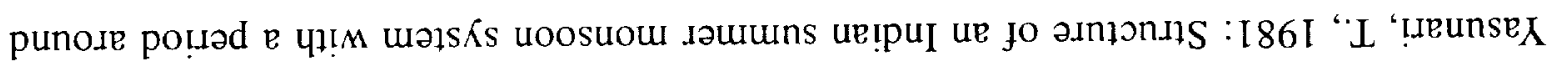

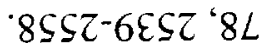

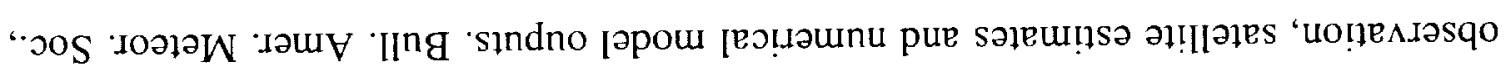

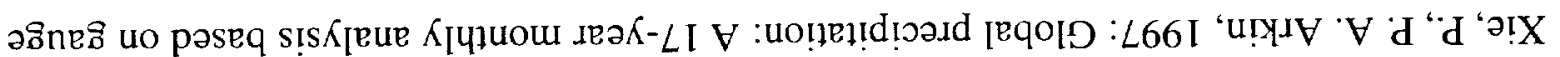

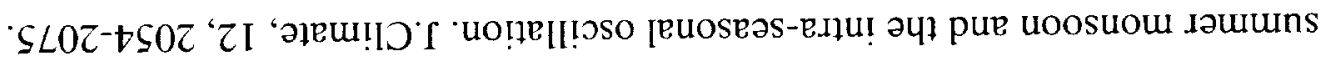

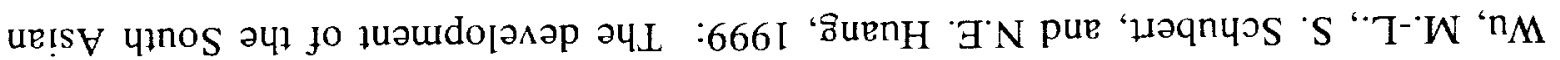

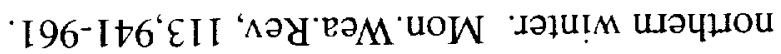

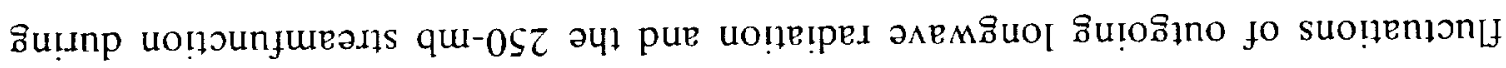

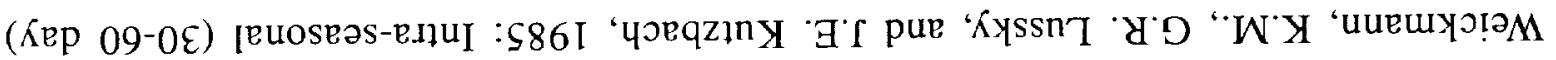

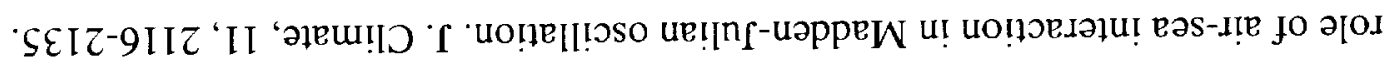

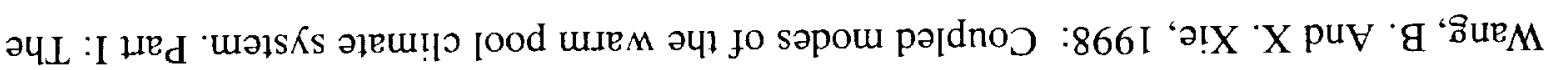
'ssard u! " "IOS 'soult

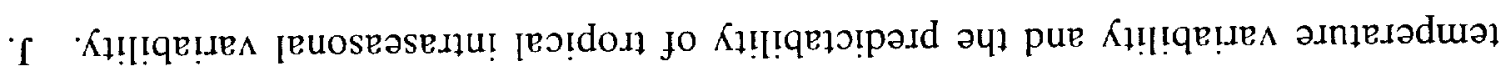

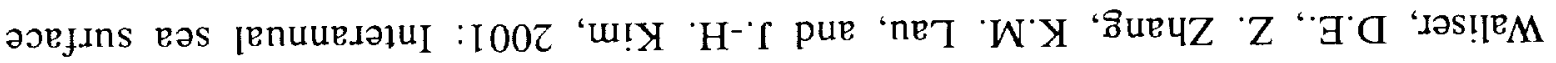
$8 S \mathcal{E}-\varepsilon \varepsilon \mathcal{E}$ '9S "!

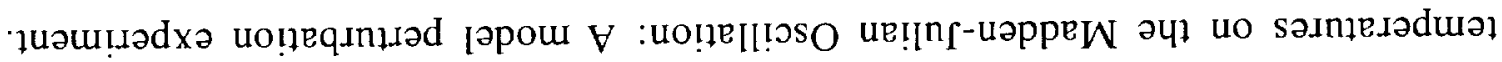

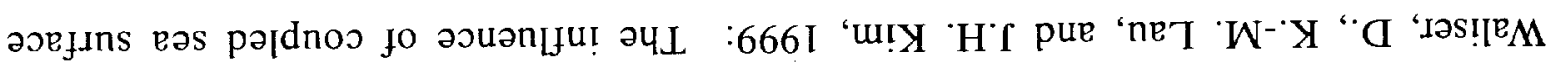

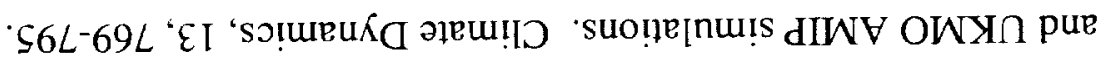

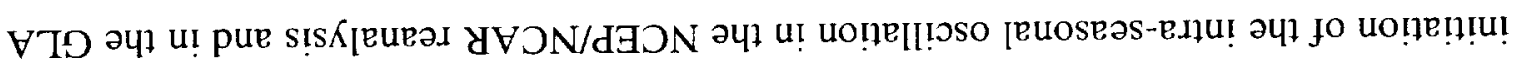




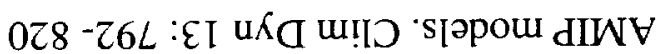

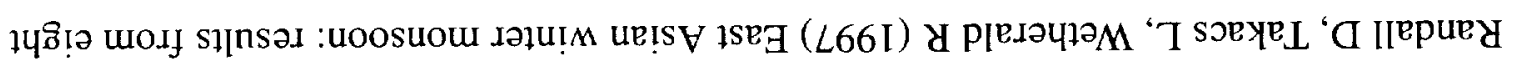

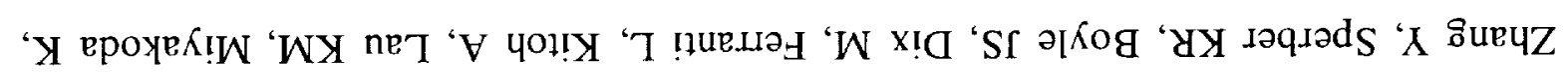

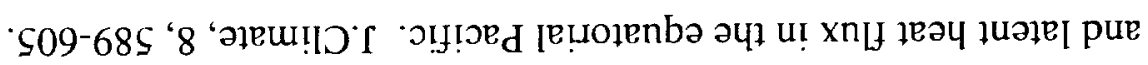

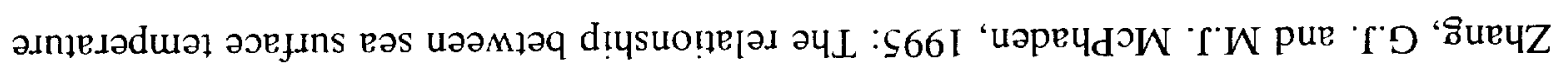



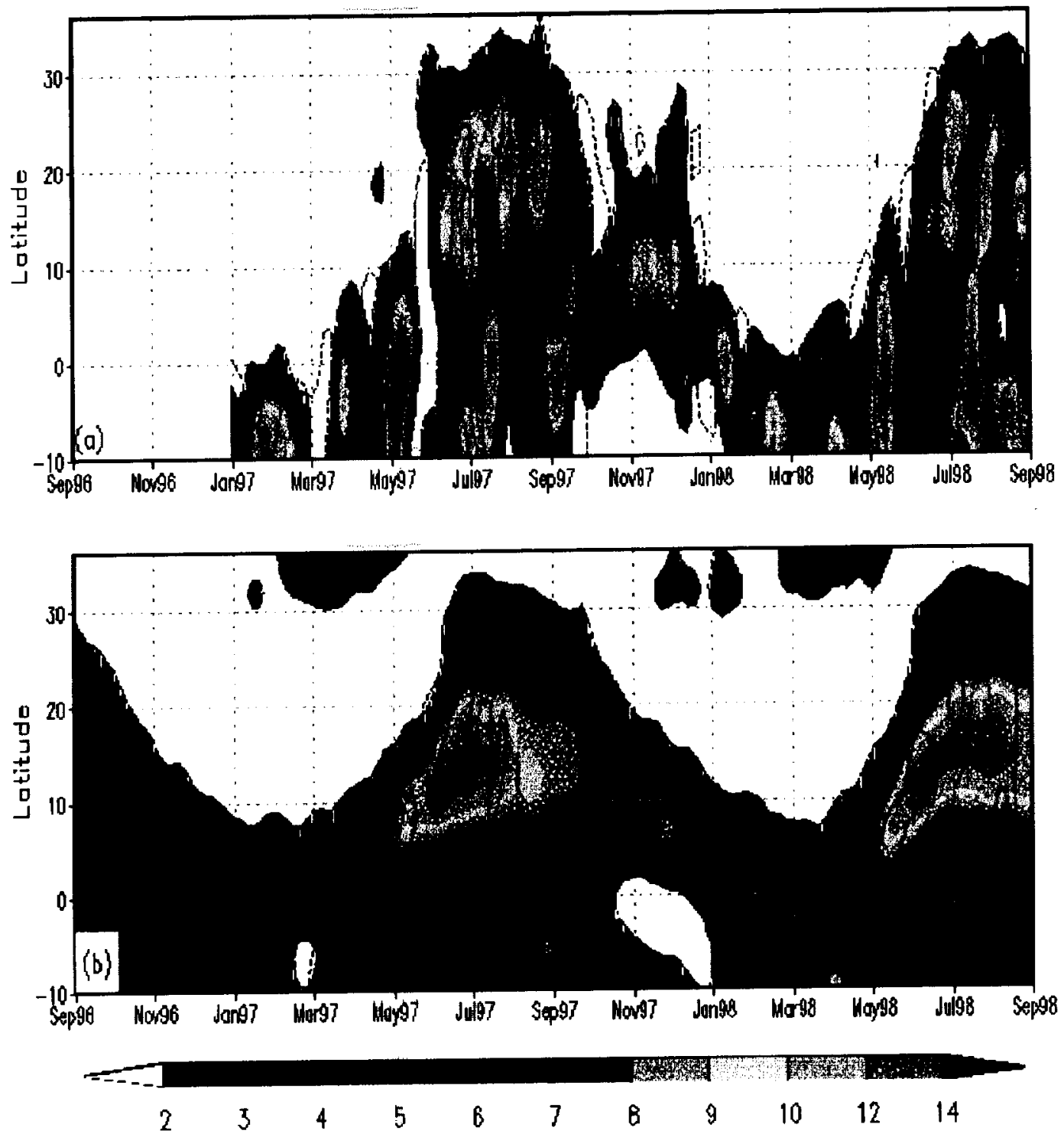

Fig. 1: a) Latitude-time section of the observed daily precipitation ( $\mathrm{mm} /$ day) averaged between $75^{\circ} \mathrm{E}-85^{\circ} \mathrm{E}$. Values are only available beginning January 1997 . The shading denotes the total precipitation and the contours denote the precipitation filtered to retain time scales between 20 and 70 days. b) Same as (a), but for the ensemble and model mean precipitation. For the filtered data we outline in (a) the $+/-1 \mathrm{~mm} /$ day values, and in (b) the $+/-0.5 \mathrm{~mm} /$ day values. 


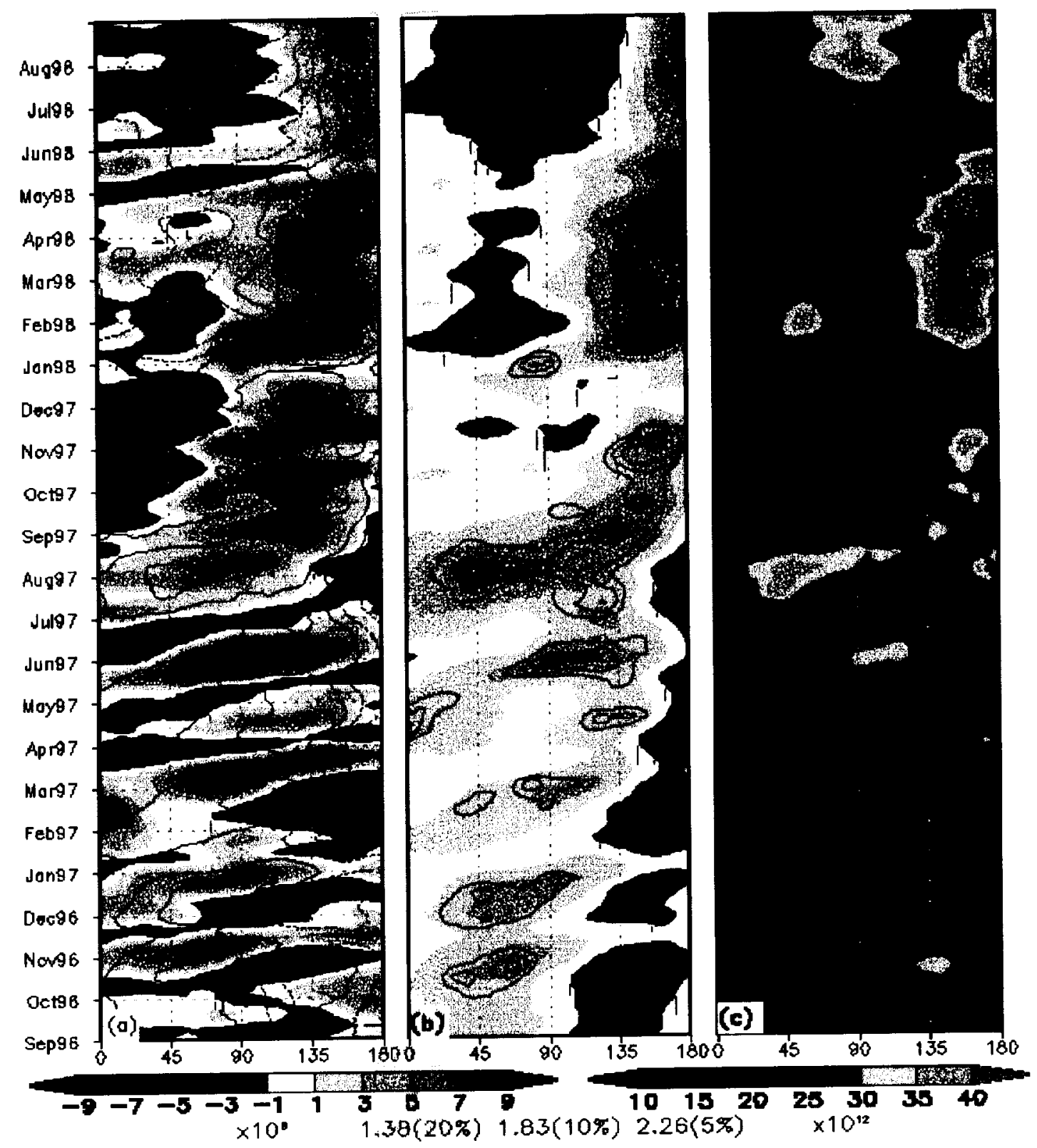

Fig. 2: Left panel: The colored field is a longitude-time section of the NCEP/NCAR reanalysis $200 \mathrm{mb}$ velocity potential unfiltered pentad anomalies averaged between $5^{\circ} \mathrm{N}$ and $10^{\circ} \mathrm{N}$. The contours are the same quantity, except for the ensemble and model mean anomaly fields. Center panel: The colored field is a repeat of the ensemble and model mean anomaly fields with superimposed contours of the 3 significance levels indicated at the bottom of the figure. Right panel: The intra-ensemble variance (averaged over all models) of the filtered $(20-70$ day) $200 \mathrm{mb}$ velocity potential anomalies averaged between $5^{\circ} \mathrm{N}$ and $10^{\circ} \mathrm{N}$. Units: $\mathrm{m}^{2} / \mathrm{s}$ for the mean and $\left(\mathrm{m}^{2} / \mathrm{s}\right)^{2}$ for the variance. 


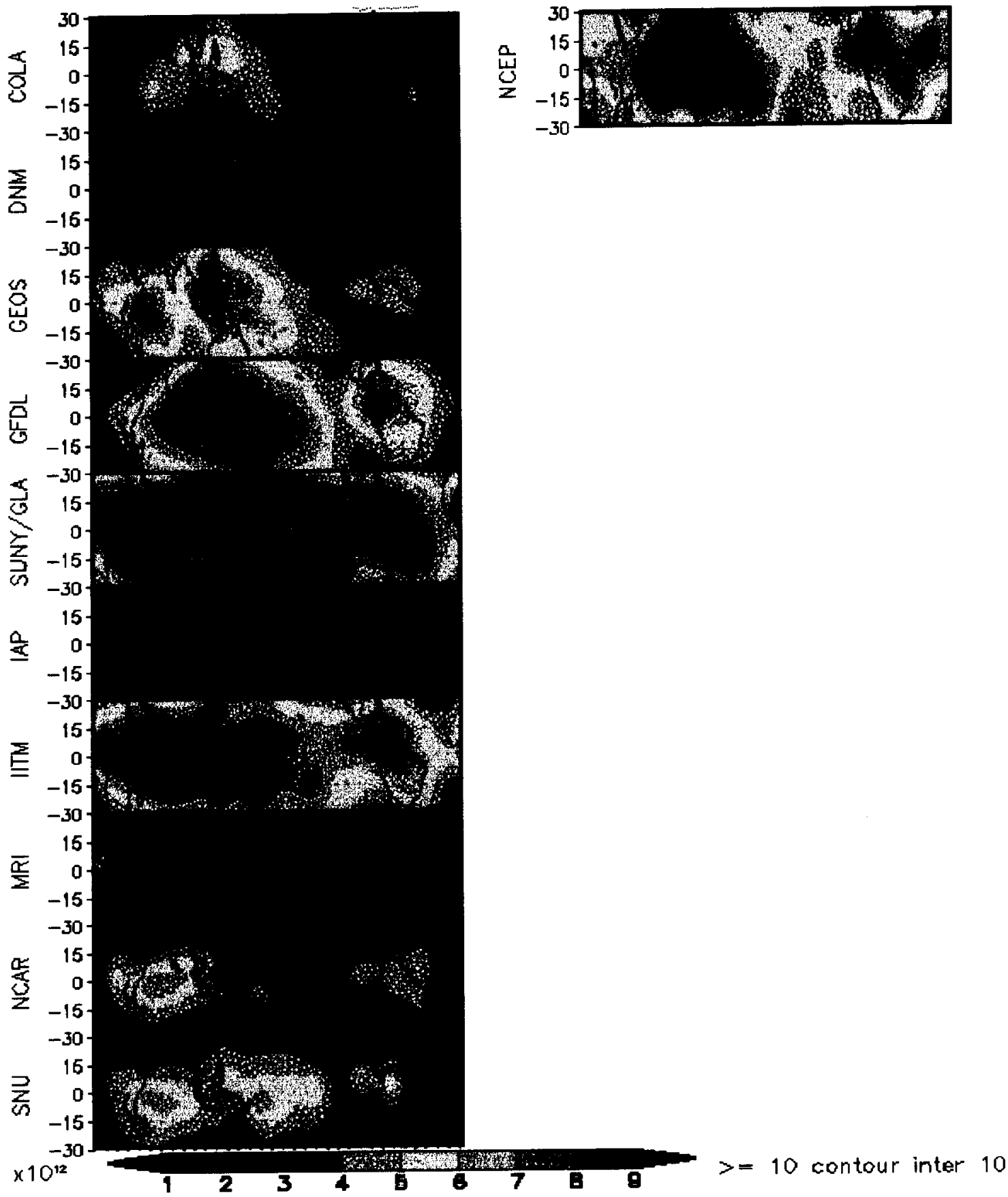

Fig. 3: The total variance of the filtered (20-70 day) $200 \mathrm{mb}$ velocity potential for each model and the NCEP/NCAR reanalysis for the time period September 1996 through August 1998. Units are: $\mathrm{m}^{4} \mathrm{~s}^{2}$ 


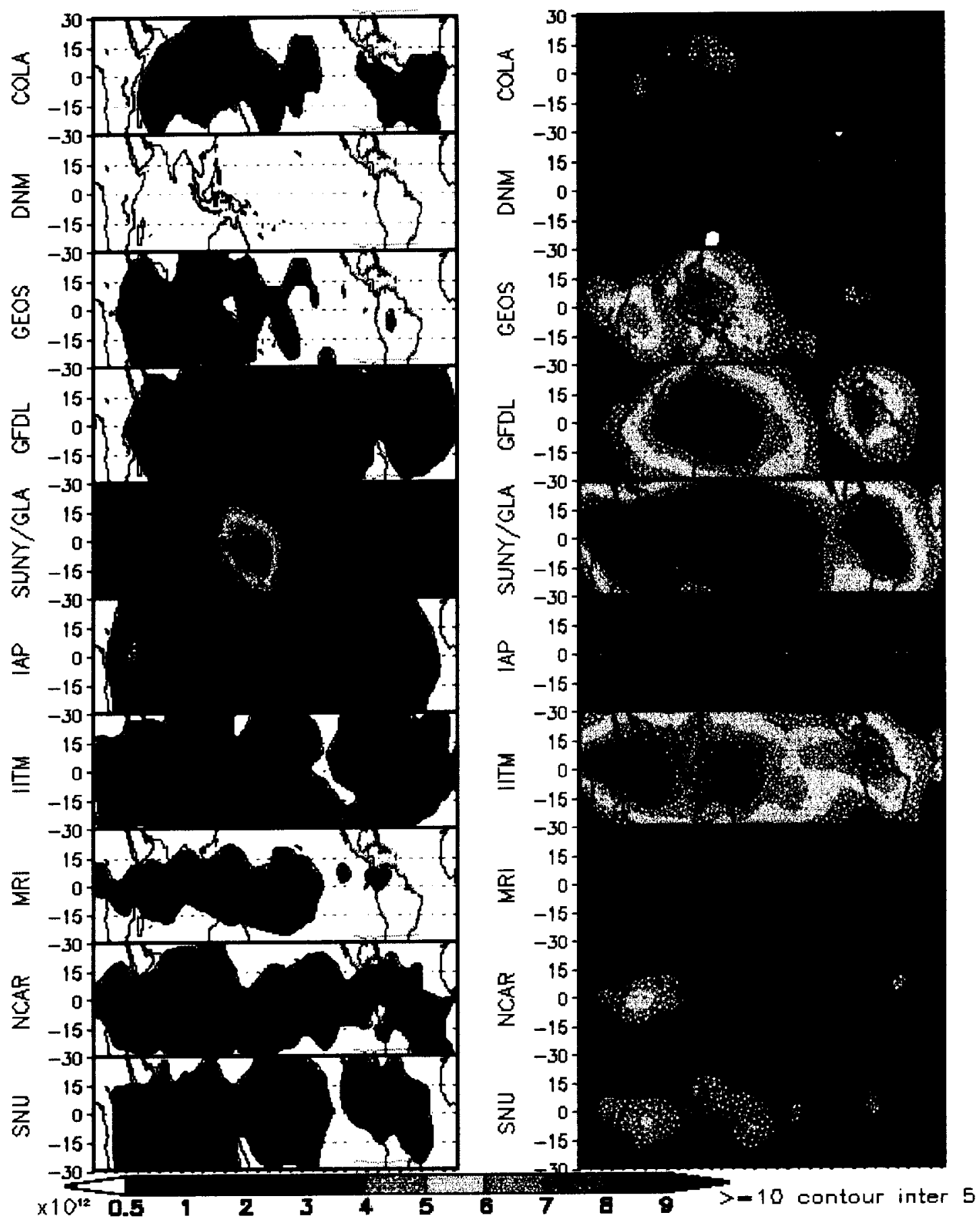

Fig. 4: Left panel: The forced (inter-ensemble) variance of the filtered (20-70 day) $200 \mathrm{mb}$ velocity potential for each model. Right panel: The free (intra-ensemble) variance of the filtered (20-70 day) $200 \mathrm{mb}$ velocity potential for each model. Units are: $\mathrm{m}^{4} \mathrm{~s}^{2}$ 


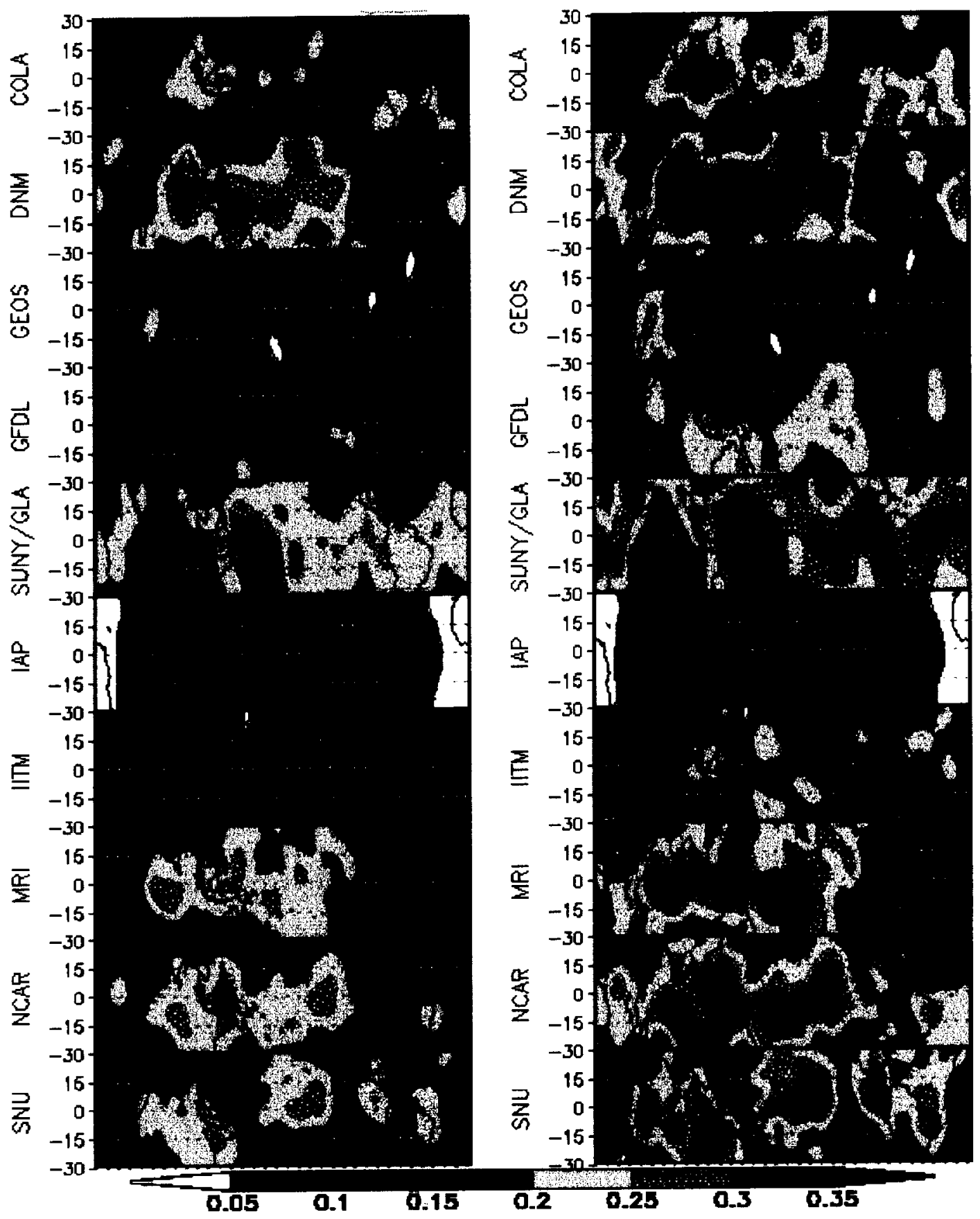

Fig. 5: Left panel: The ratio of the forced (inter-ensemble) to total variance of the filtered (20-70 day) $200 \mathrm{mb}$ velocity potential for each model $\left(P_{\beta}\right)$. Right panel: Same as the left panel but for the ratio of the forced (inter-ensemble) to free (intra-ensemble) variance $(U)$. 


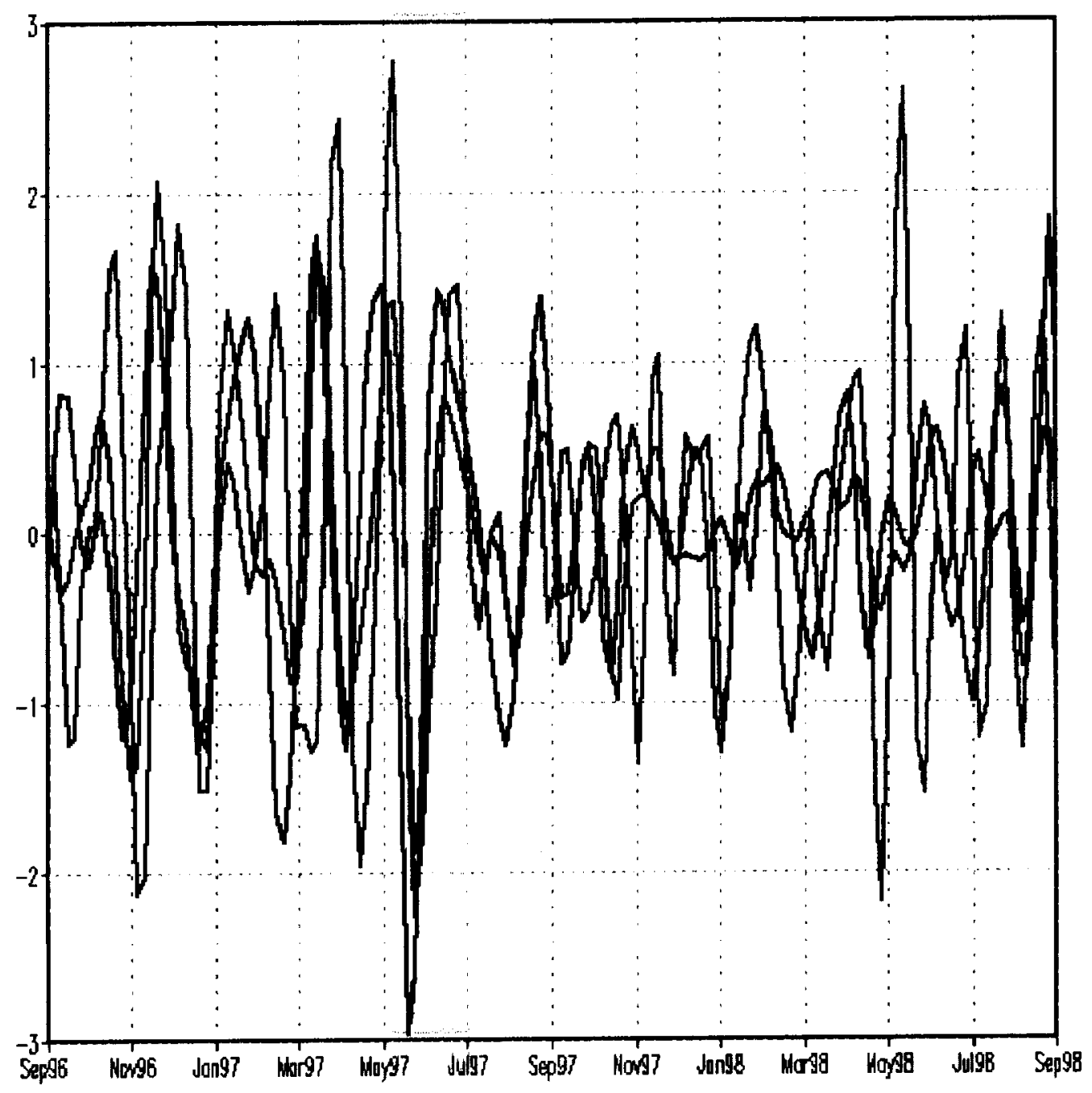

Fig. 6: Time series of the $20-70$ day filtered $200 \mathrm{mb}$ velocity potential (multiplied by minus one) and SST anomalies averaged from $60^{\circ} \mathrm{E}-100^{\circ} \mathrm{E}$ and $10^{\circ} \mathrm{S}-10^{\circ} \mathrm{N}$. The red curve is the ensemble and model mean velocity potential. Multiply values by $1.0 \times 10^{6}$ to get units of $\mathrm{m}^{2} / \mathrm{s}$. The blue curve is the NCEP/NCAR reanalysis velocity potential. Multiply values by $3.3 \times 10^{6}$ to get units of $\mathrm{m}^{2} / \mathrm{s}$. The black curve is the SST in units of $0.1^{\circ} \mathrm{C}$. 


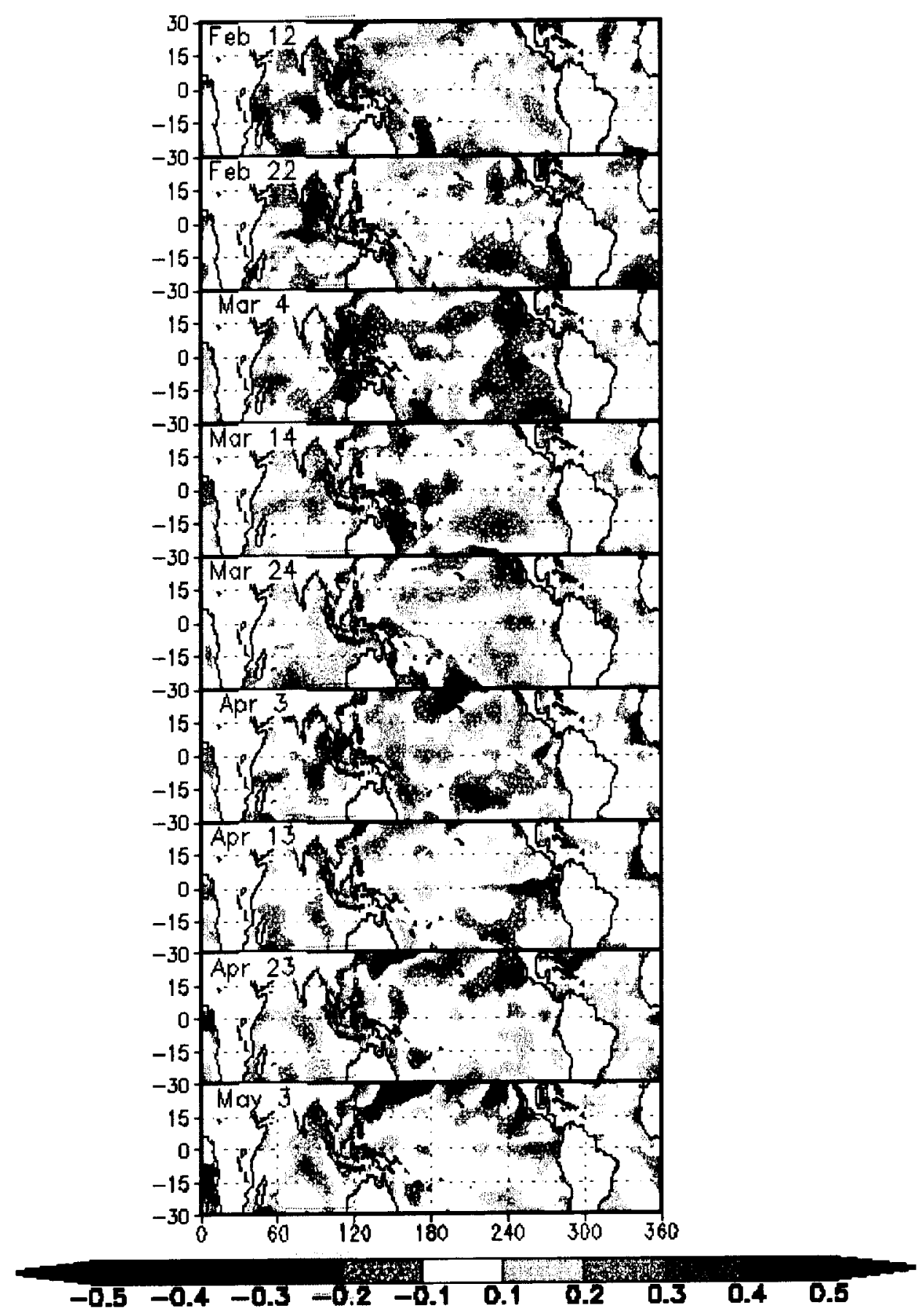

Fig. 7: Ten day averages of the filtered (20-70 day) SST anomalies for the period February 12 through May 12,1997 . Units are: ${ }^{\circ} \mathrm{C}$. 


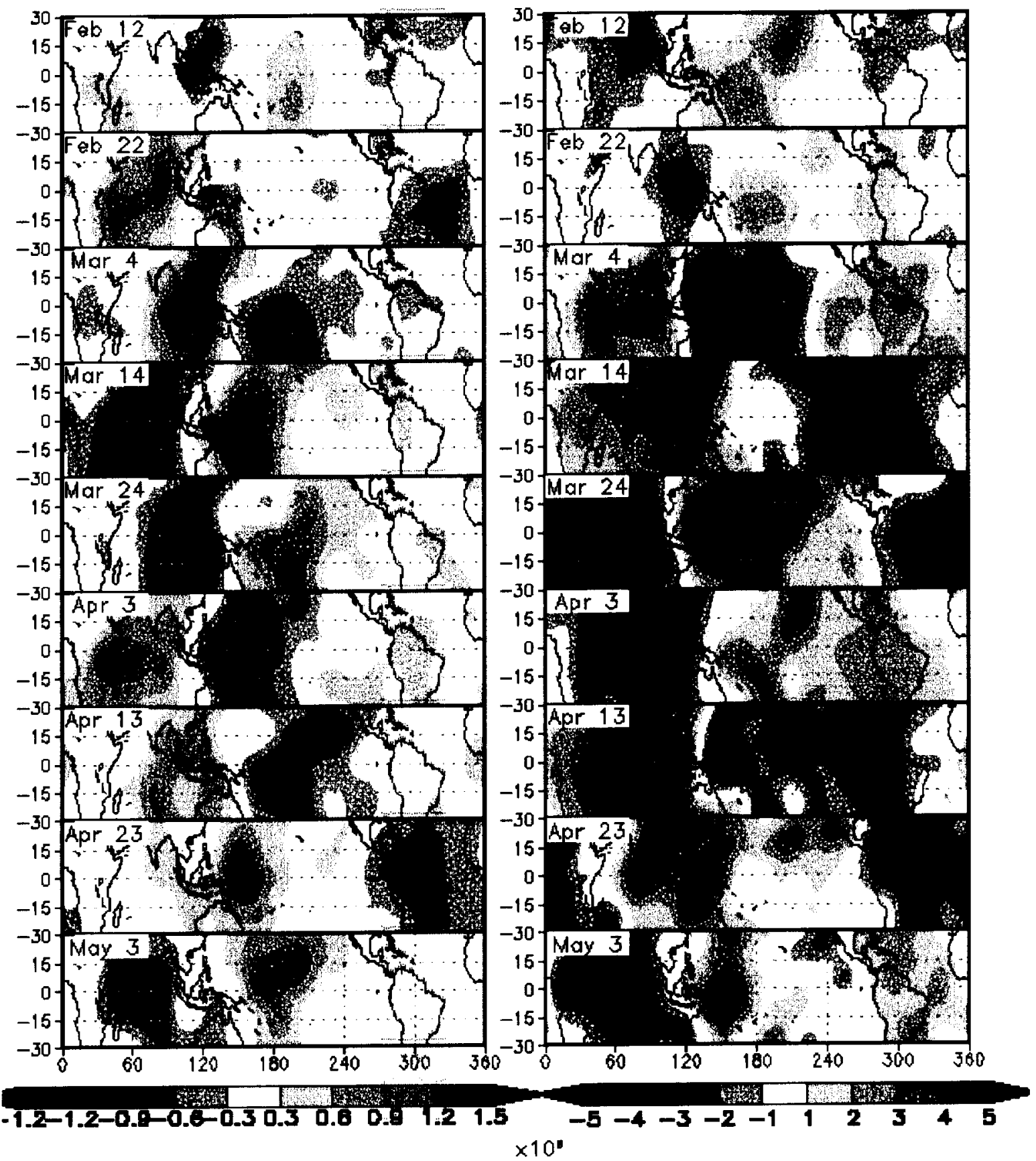

Fig. 8: Left panel: Ten day averages of the filtered (20-70 day) ensemble and model mean $200 \mathrm{mb}$ velocity potential field for the period February 12 through May 12, 1997. Right panel: Ten day averages of the NCEP/NCAR reanalysis filtered (20-70 day) $200 \mathrm{mb}$ velocity potential anomalies for the period February 12 through May 12, 1997. The reanalysis anomalies are computed as deviations from the 1982-98 climatology of 10 day averages. Units are: $\mathrm{m}^{2} \mathrm{~s}^{-1}$. 

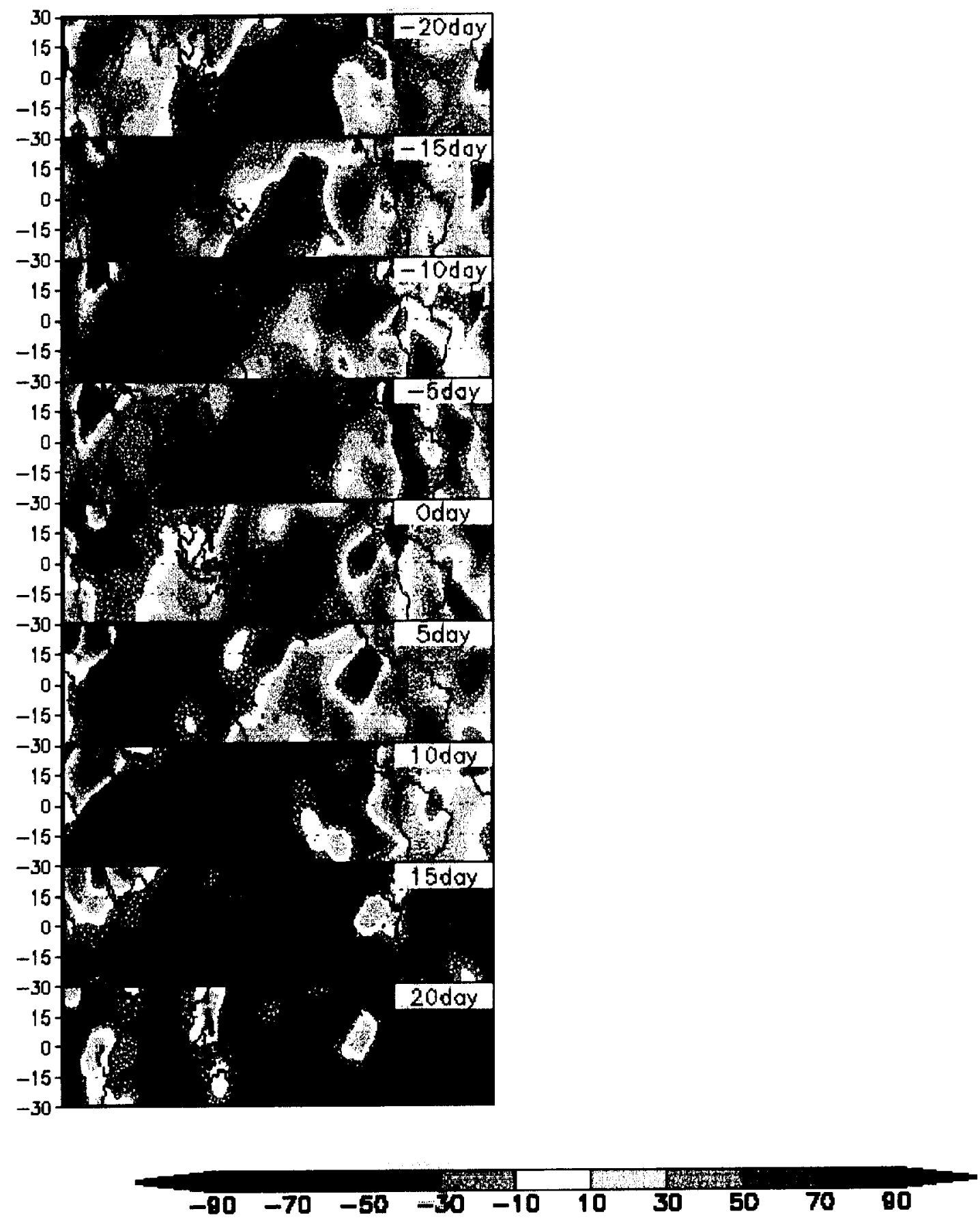

Fig. 9: Lag correlations between the filtered (20-70 day) ensemble/model mean and the observed (reanalysis) $200 \mathrm{mb}$ velocity potential anomalies computed over for the period February 12 through May 12,1997. Negative lags indicate the simulated anomalies lead the observed anomalies. 

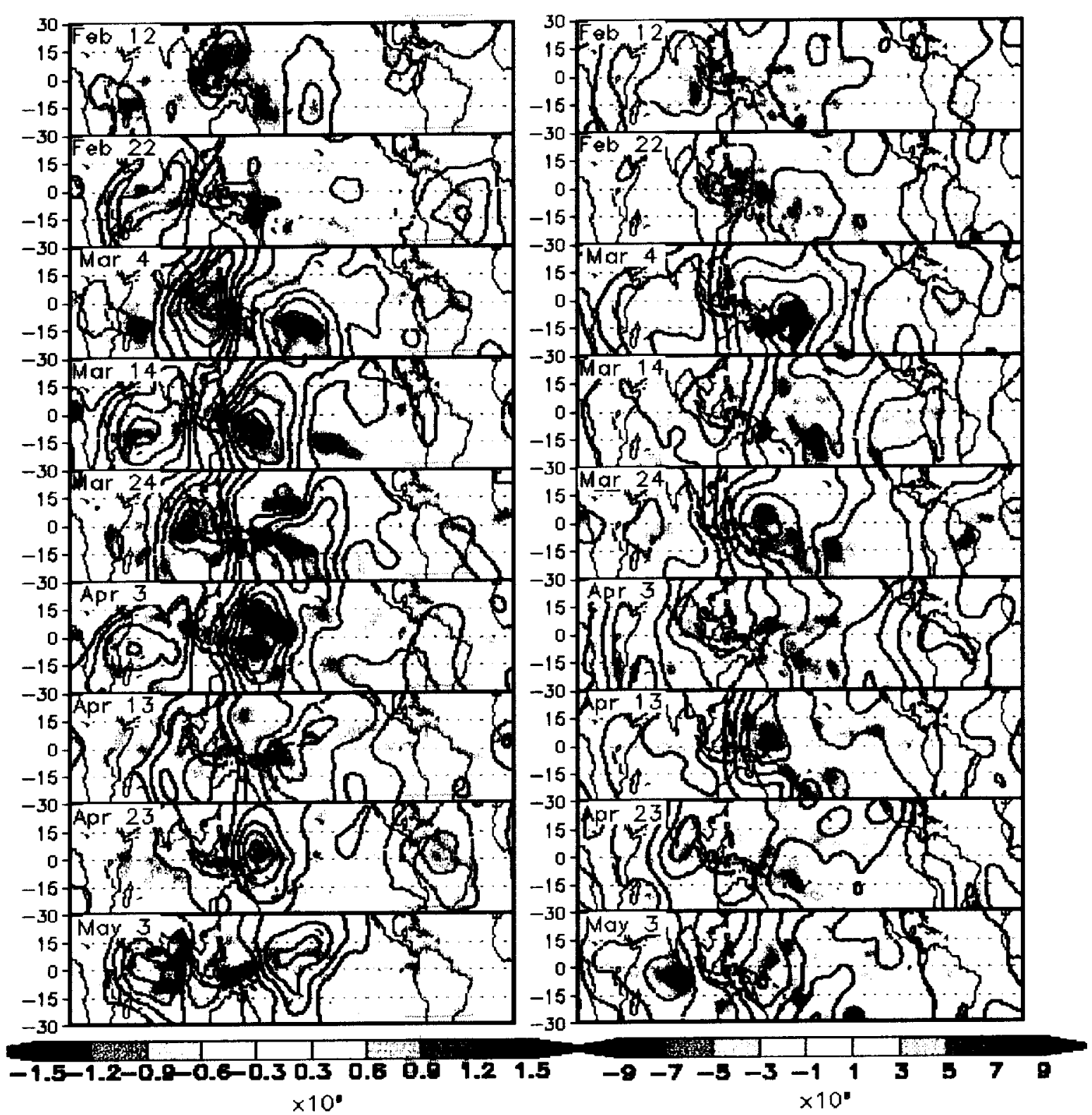

Fig. 10: Same as Fig. 8, but for precipitation (shading) and a repeat of the $200 \mathrm{mb}$ velocity potential (contours, blue are negative values). Values in the left panel are the ensemble and model mean. Values in the right panel are the GPCP precipitation and NCEP/NCAR reanalysis $200 \mathrm{mb}$ velocity potential. Units for precipitation are $\mathrm{mm} / \mathrm{day}$. Contour intervals for $200 \mathrm{mb}$ velocity potential are, for the model results $+/-0.3,0.6,0.9,1.2,1.5$ $\times 10^{6} \mathrm{~m}^{2} / \mathrm{s}$, and for the reanalysis $+1-1,3,5,7,9,11 \times 10^{6} \mathrm{~m}^{2} / \mathrm{s}$. 


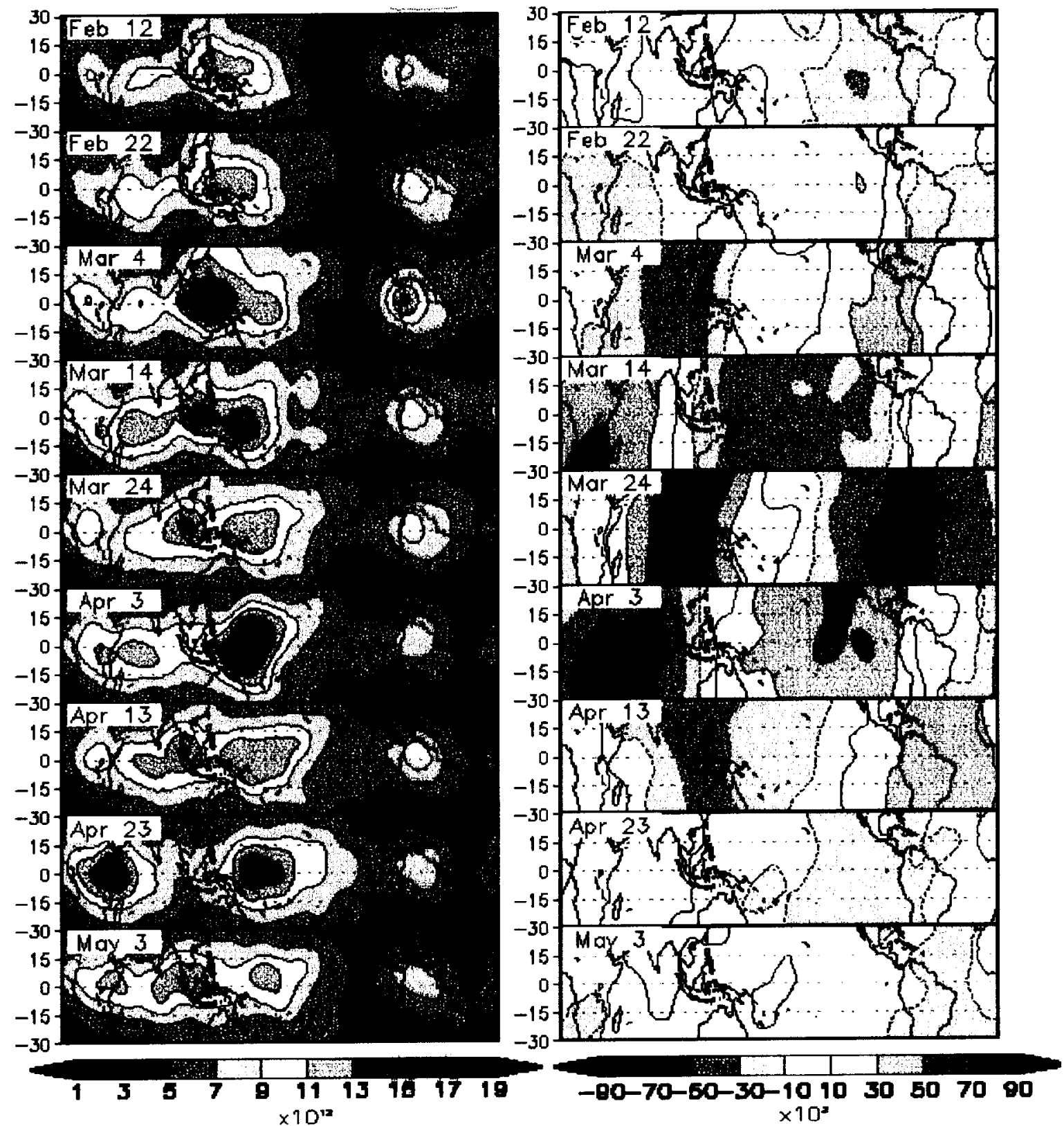

Fig. 11: Left panel: The intra-ensemble variance of the $200 \mathrm{mb}$ velocity potential averaged over all models. Right panel: The time lag correlations (x100) of the $200 \mathrm{mb}$ velocity potential. The base period is the 10-day average period March 24-April 2, 1997 (center panel). The base region is the area-average of $\left(80^{\circ} \mathrm{E}-110^{\circ} \mathrm{E}, 15^{\circ} \mathrm{S}-10^{\circ} \mathrm{N}\right)$. The lag correlations are computed between the base region and all other grid points and 10-day averaged time lags $(+/-0,10,20,30,40$ days $)$ over all ensemble members and models. See text for details. 


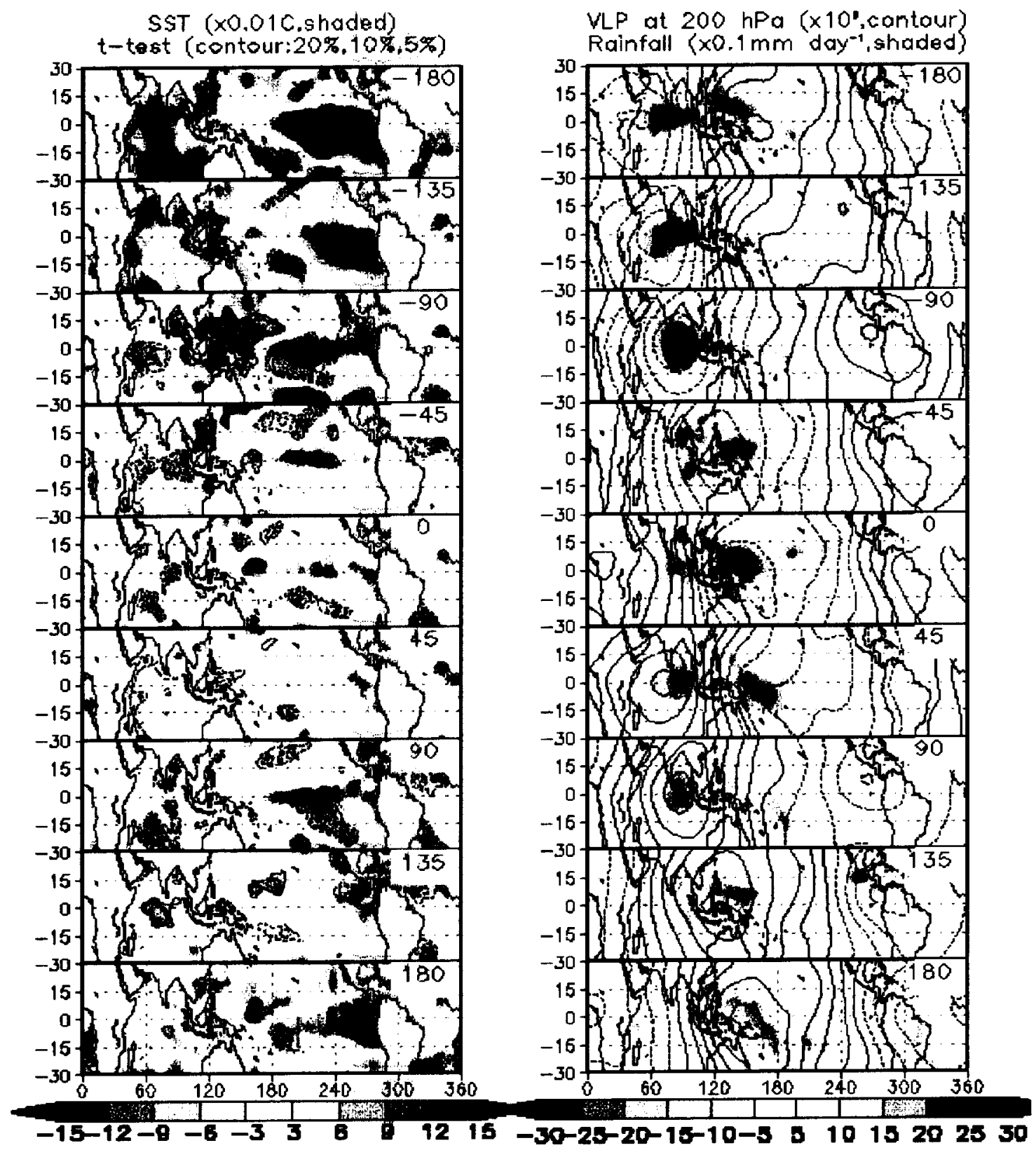

Fig. 12: The composite evolution of the MJO using the leading complex EOF of the filtered (20-70day) NCEP/NCAR reanalysis $200 \mathrm{mb}$ velocity potential field as an index. The composite evolution goes through a complete cycle $\left(-180^{\circ}\right.$ to +180 degrees phase). The results are based on the period 1982-1998: values are included in the composite for only those times when the amplitude of the leading EOF is greater than one standard deviation. Left panel: SST (shaded) with significance values contoured as indicated. Right panel: GPCP precipitation (shaded) and $200 \mathrm{mb}$ velocity potential (contours). Units for velocity potential are: $\mathrm{m}^{2} / \mathrm{s}$. 


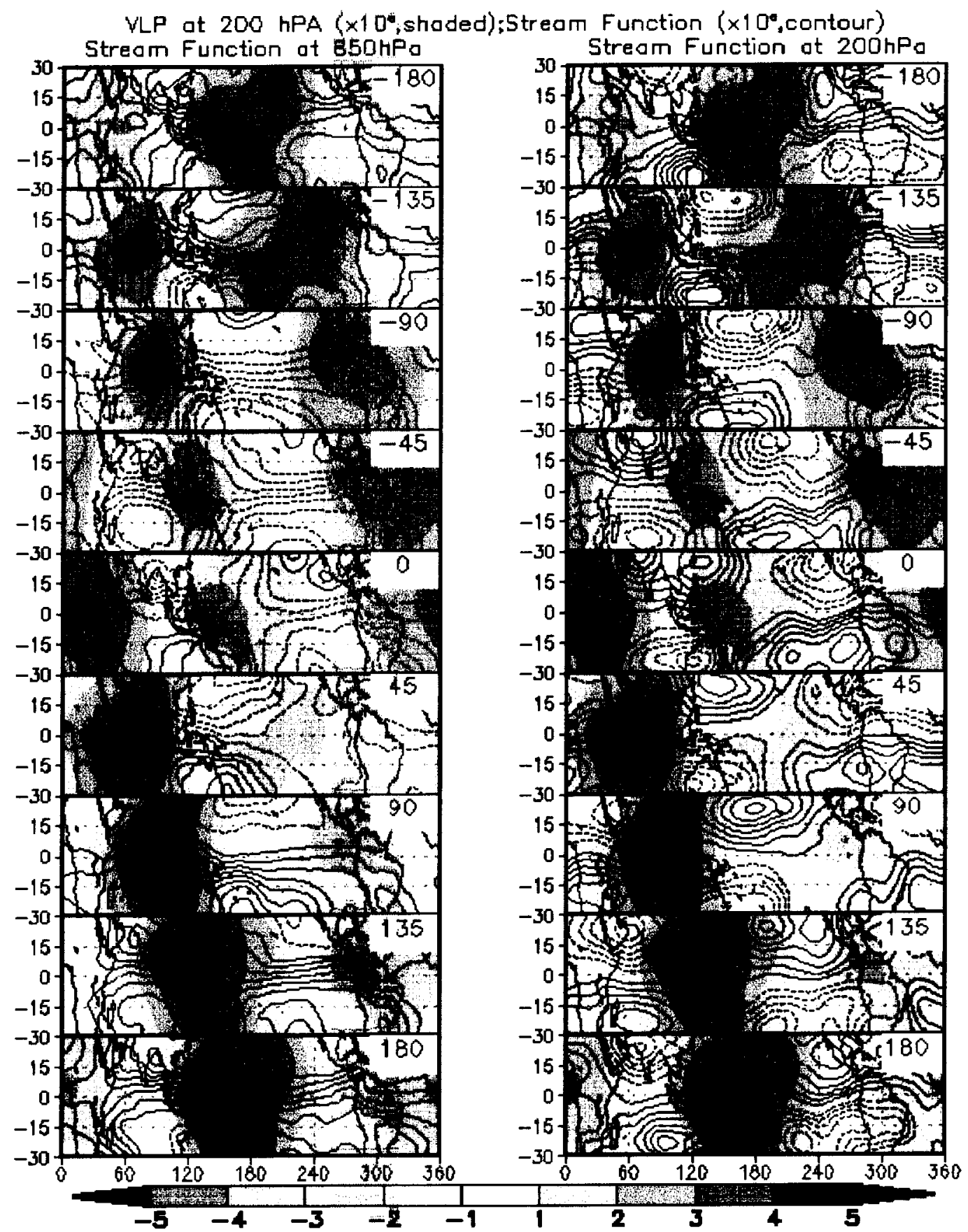

Fig. 13: Same as Fig. 12, but for the NCEP/NCAR reanalysis stream function and velocity potential. Left panel: $850 \mathrm{mb}$ stream function (contours) and $200 \mathrm{mb}$ velocity potential (shaded). Right panel: $200 \mathrm{mb}$ stream function (contours) and $200 \mathrm{mb}$ velocity potential (shaded). Units for stream function and velocity potential are: $\mathrm{m}^{2} / \mathrm{s}$. 

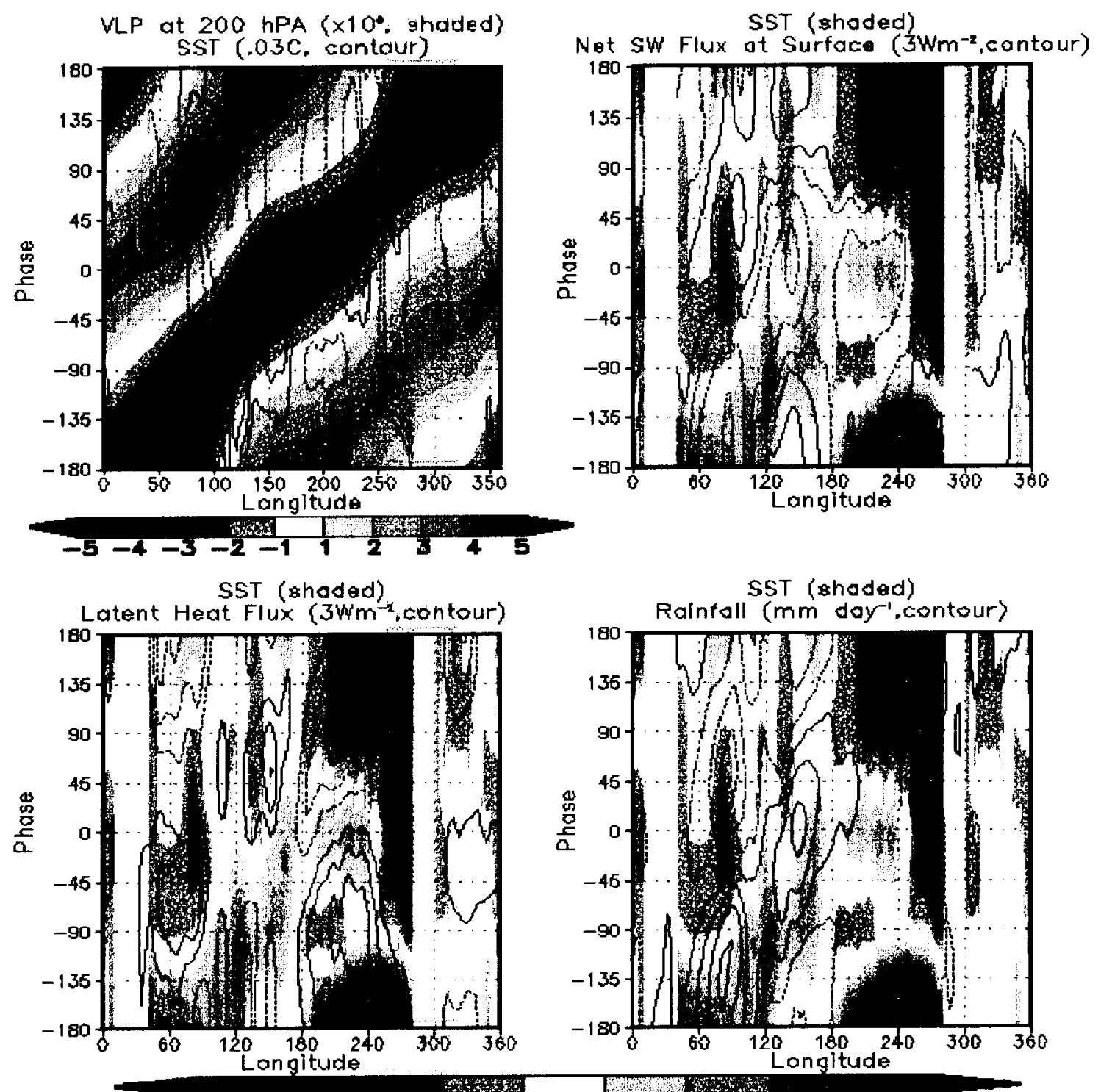

$\begin{array}{llllllll}-0.16-0.12-0.09 & -0.65-0.03 & 0.03 & 0.06 & 0.06 & 0.12 & 0.16\end{array}$

Fig. 14: Same as Fig. 12, but for phase-longitude diagrams for various fields averaged between $6^{\circ} \mathrm{S}$ and $6^{\circ} \mathrm{N}$. Top left panel is the $200 \mathrm{mb}$ velocity potential (shaded) and the SST (contours start at $+/-0.03$ ). Units for velocity potential are: $\mathrm{m}^{2} / \mathrm{s}$. Top right panel is the SST (shaded) and the NCEP/NCAR reanalysis net surface short wave flux at the surface (contours start at $+/-1$ ). Bottom left panel is the SST (shaded) and NCEP/NCAR latent heat flux (contours start at $+1-3$ ). Bottom right panel is the SST (shaded) and the observed precipitation (contours start at $+/-0.5$ ). The bottom color bar refers to the SST fields. 